\title{
Zimbabwe: Statistical Appendix
}

This Statistical Appendix for Zimbabwe was prepared by a staff team of the International Monetary Fund as background documentation for the periodic consultation with the member country. It is based on the information available at the time it was completed on June 24, 2004. The views expressed in this document are those of the staff team and do not necessarily reflect the views of the government of Zimbabwe or the Executive Board of the IMF.

The policy of publication of staff reports and other documents by the IMF allows for the deletion of market-sensitive information.

To assist the IMF in evaluating the publication policy, reader comments are invited and may be sent by e-mail to publicationpolicy@imf.org.

Copies of this report are available to the public from

International Monetary Fund • Publication Services

700 19th Street, N.W. • Washington, D.C. 20431

Telephone: (202) 6237430 • Telefax: (202) 6237201

E-mail: publications@imf.org • Internet: http://www.imf.org

Price: $\$ 15.00$ a copy

\section{International Monetary Fund Washington, D.C.}





\title{
INTERNATIONAL MONETARY FUND
}

\author{
ZIMBABWE
}

\section{Statistical Appendix}

Prepared by African Department

Prepared by a staff team consisting of Doris C. Ross (head), Jean-François Dauphin, Faisal Ahmed, Ashwini Jayaratnam (all AFR), Helga Treichel (FIN),

Padamja Khandelwal (PDR), and Dmitriy Rozhkov (MFD)

Approved by African Department

June 24, 2004

Contents

Page

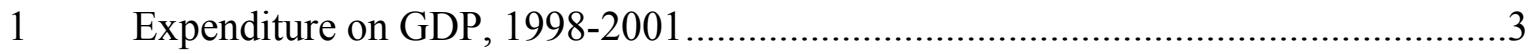

2. Gross Domestic Product, 1998-2003 ……………..............................................

3. Agricultural Crop Production, 1998-2003 ………………………………….....

4. Prices of Marketed Agricultural Crops, 1997/98-2002/03 ........................................6

5. Area Under Cultivation for Major Crops, 1998-2003 …………………………......

6. Volume and Value of Livestock Slaughtering and Milk Production, 1998-2003 .....8

7. Livestock in Communal and Commercial Farming Areas, 1998-2002 …….............9

8. Volume of Manufacturing Output, 1998-2003 ………........................................10

9. Mineral Production, 1998-2003 …………………..........................................11

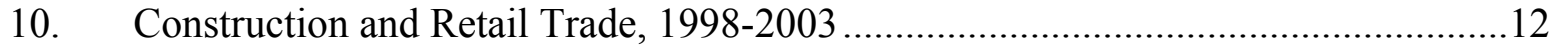

11. Electrical Energy Produced and Distributed, 1998-2003 .........................................13

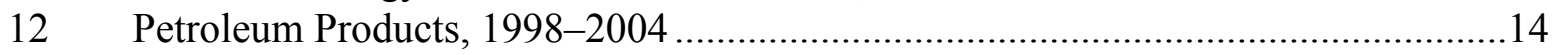

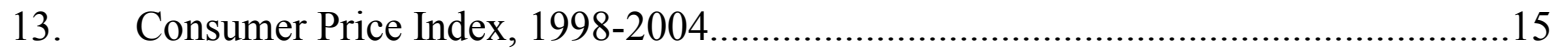

14. Consumer Price Index, December 2002-April 2004 ……………………...............16

15. Employment and Employment Earnings, 1998-2002 ……...................................17

16. Central Government Operations, 1999-2003 ……...............................................18

17. Detailed Central Government Revenue, 1999-2003 …………................................19

18. Detailed Central Government Expenditure and Net Lending, 1999-2003 _...............20

19. Expenditure and Repayments by Ministries, 1999-2003 ..........................................21

20. Civil Service Employment Budgeted Posts, 1999-2003 ………...............................22

21. Central Government Debt and Debt-Service Payments, 1999-2003 ……................23

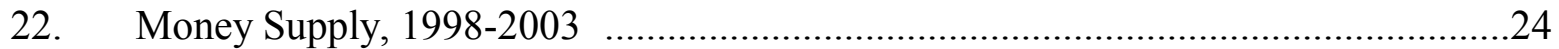

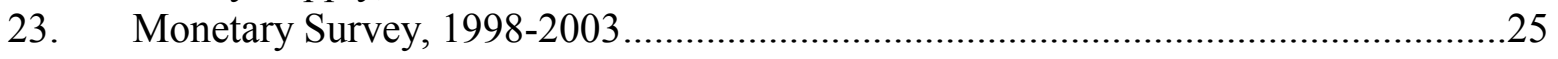

24. Assets and Liabilities of Monetary Authorities, 1998-2003 ………………….......26 
Content

25. Zimbabwe: Consolidated Accounts of Deposit Money Banks and Other Banking Institutions, 1998-2003 …...............................................................27

26. Required Reserves and Liquid Asset Ratios, 1998-2003 …................................28

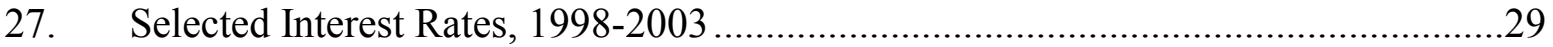

28. Sectoral Analysis of Commercial Banks' Loans and Advances, 1998-2003 .............30

29. Sectoral Analysis of Merchant Banks' Loans and Advances, 1998-2002 .................31

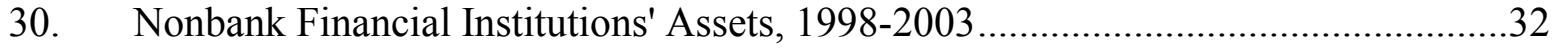

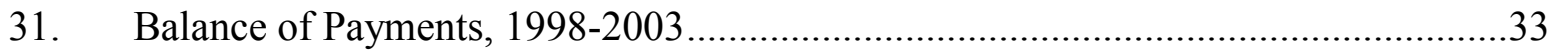

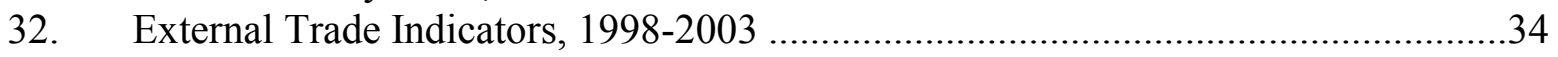

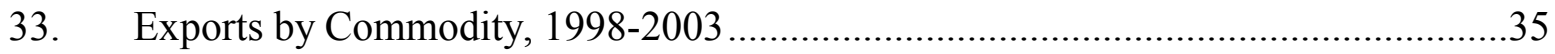

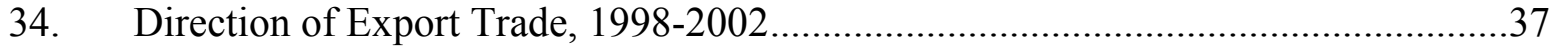

35. Imports by Principal Commodities, 1998-2003 …..................................................38

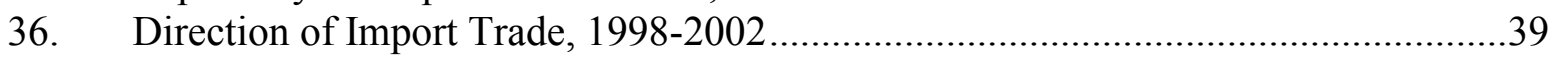

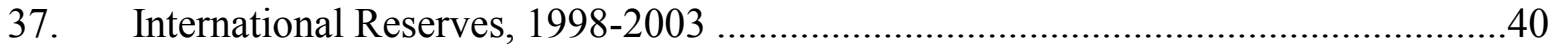

38. External Debt Outstanding By Creditors, 1998-2003 .......................................41

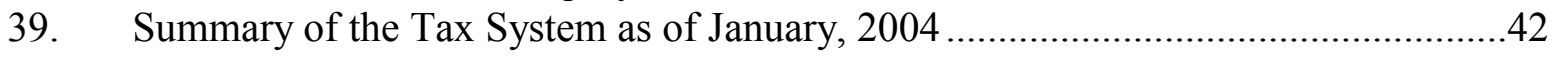


Table 1. Zimbabwe: Expenditure on GDP, 1998-2001 ${ }^{1 /}$

\begin{tabular}{|c|c|c|c|c|}
\hline & 1998 & 1999 & 2000 & 2001 \\
\hline & \multicolumn{4}{|c|}{ (In millions of Zimbabwe dollars) } \\
\hline Consumption & 116,320 & 191,817 & 283,490 & 578,043 \\
\hline Private & 93,647 & 160,849 & 237,645 & 517,905 \\
\hline Central government & 22,673 & 30,968 & 45,845 & 60,138 \\
\hline Gross fixed capital formation & 29,593 & 30,272 & 40,237 & 30,531 \\
\hline Government & 2,458 & 3,862 & 3,862 & $\ldots$ \\
\hline Private & 27,135 & 26,410 & 36,375 & $\ldots$ \\
\hline Change in stocks & 214 & 2,614 & 5,822 & $-12,301$ \\
\hline Gross investment & 29,807 & 32,886 & 46,059 & 18,230 \\
\hline Net exports of goods and nonfactor services & $-2,482$ & 3,731 & -891 & $-31,633$ \\
\hline Exports & 62,332 & 105,977 & 118,140 & 105,335 \\
\hline Imports & $-64,814$ & $-102,246$ & $-119,031$ & $-136,968$ \\
\hline GDP (at market prices) & 143,645 & 228,434 & 328,658 & 564,640 \\
\hline Net factor income from abroad & $-9,155$ & $-9,431$ & $-11,286$ & $-11,935$ \\
\hline Factor income received from abroad & 414 & 1,491 & 1,268 & 755 \\
\hline Factor income paid abroad & $-9,569$ & $-10,922$ & $-12,554$ & $-12,690$ \\
\hline \multirow[t]{2}{*}{ Gross national income } & 134,490 & 219,003 & 317,372 & 552,705 \\
\hline & \multicolumn{4}{|c|}{ (In percent of GDP) } \\
\hline National savings, excluding grants & 12.6 & 12.3 & 10.7 & -4.6 \\
\hline Government & -2.0 & -5.9 & $\ldots$ & $\ldots$ \\
\hline Private & 14.6 & 18.2 & $\ldots$ & $\ldots$ \\
\hline Gross fixed capital formation & 20.6 & 13.3 & 12.2 & 8.2 \\
\hline Government & 1.7 & 1.7 & 1.2 & $\ldots$ \\
\hline Private & 18.9 & 11.6 & 11.1 & $\ldots$ \\
\hline Change in stocks & 0.1 & 1.1 & 1.8 & -2.2 \\
\hline Gross investment & 20.8 & 14.4 & 14.0 & 6.0 \\
\hline
\end{tabular}

Source: Central Statistical Office.

1/ Data for 2002 and 2003 are not available. Estimates for nominal GDP at market prices are Z\$1,205 and Z\$5,810 billion respectively. 
Table 2. Zimbabwe: Gross Domestic Product, 1998-2003

(Percent change at constant 1990 prices)

\begin{tabular}{|c|c|c|c|c|c|c|}
\hline & 1998 & 1999 & 2000 & 2001 & 2002 & 2003 \\
\hline Agriculture, hunting, fishing, and forestry & 2.3 & 3.6 & 3.2 & -3.4 & -18.5 & -8.0 \\
\hline Mining and quarrying & 8.4 & -7.0 & -6.9 & -13.5 & 11.4 & -9.8 \\
\hline Manufacturing & -3.4 & -4.5 & -11.5 & -5.4 & -16.4 & -12.5 \\
\hline Electricity and water & -4.3 & 7.6 & -0.6 & 10.0 & 1.0 & 1.1 \\
\hline Construction & 6.0 & -11.1 & -15.0 & -35.2 & -10.1 & -9.5 \\
\hline Finance and insurance & 6.2 & -4.5 & 0.8 & -1.2 & 1.0 & 1.0 \\
\hline Real estate & 5.4 & 5.1 & 4.9 & 4.1 & 5.0 & 1.0 \\
\hline Distribution, hotels, and restaurants & 0.8 & 0.4 & -8.8 & -5.2 & -15.5 & -12.5 \\
\hline Transport and communications & -11.0 & -3.7 & -7.5 & -5.9 & -7.5 & -13.5 \\
\hline Public administration & -3.0 & -4.8 & -6.2 & 5.4 & -8.0 & -8.5 \\
\hline Education & 6.8 & -8.0 & 3.5 & 5.9 & -8.5 & -7.8 \\
\hline Health & 6.6 & 14.5 & -7.8 & 17.9 & -10.0 & -12.4 \\
\hline Domestic services & -1.1 & -6.3 & -5.2 & 5.8 & -3.0 & -3.1 \\
\hline Other services & 2.2 & 7.4 & -2.1 & -2.8 & -2.2 & -2.5 \\
\hline Less: imputed bank service charges & 37.4 & 13.9 & 25.5 & 3.0 & -3.3 & 1.0 \\
\hline GDP (at factor cost) & -0.8 & -2.1 & -6.1 & -3.6 & -10.8 & -8.9 \\
\hline Net other taxes on production & 39.4 & -16.2 & -16.9 & -24.6 & -13.5 & -2.5 \\
\hline GDP at basic prices & -0.7 & -2.2 & -6.1 & -3.6 & -10.8 & -8.9 \\
\hline Net taxes on products & 10.8 & -14.7 & -24.0 & 6.7 & -13.9 & -25.0 \\
\hline GDP at market prices & 0.5 & -3.6 & -7.9 & -2.8 & -11.1 & -9.3 \\
\hline \multicolumn{7}{|l|}{ Memorandum item: } \\
\hline Real GDP (in millions of 1990 Zimbabwe dollars) & 25,520 & 24,602 & 22,665 & 22,033 & 19,590 & 17,764 \\
\hline
\end{tabular}

Sources: Central Statistical Office, 1998-2001; Reserve Bank of Zimbabwe; and IMF staff estimates, 2002-03. 
Table 3. Zimbabwe: Agricultural Crop Production, 1998-2003 ${ }^{1 /}$ (In thousands of tons)

\begin{tabular}{|c|c|c|c|c|c|c|}
\hline & 1998 & 1999 & 2000 & 2001 & $\begin{array}{r}2002 \\
\text { Est. }\end{array}$ & $\begin{array}{r}2003 \\
\text { Est. }\end{array}$ \\
\hline \multicolumn{7}{|l|}{ Commercial farms ${ }^{2 /}$} \\
\hline Tobacco (flue cured) & 179 & 166 & 178 & 186 & 164 & 71 \\
\hline Maize & 573 & 662 & 681 & 532 & 200 & 211 \\
\hline Cotton $^{3 /}$ & 61 & 48 & 47 & 46 & 31 & 21 \\
\hline Sugarcane & 1,487 & 1,186 & 2,261 & 2,477 & 3,667 & $\ldots$ \\
\hline Wheat & 240 & 286 & 251 & 232 & 154 & 11 \\
\hline Groundnuts & 8 & 9 & 12 & 13 & 59 & 50 \\
\hline Tea & 16 & 14 & 9 & 9 & 22 & 6 \\
\hline Soybeans & 115 & 117 & 132 & 130 & 65 & 63 \\
\hline Coffee & 11 & 8 & 8 & 8 & 9 & 8 \\
\hline Sunflower seeds & 3 & 2 & 3 & 2 & 1 & 1 \\
\hline Rapoko & 1 & 1 & 1 & 1 & 1 & 1 \\
\hline Sorghum & 23 & 16 & 17 & 15 & 20 & 23 \\
\hline \multicolumn{7}{|l|}{ Communal lands $^{4 /}$} \\
\hline Tobacco (flue cured) & 3 & 3 & 5 & 4 & 9 & 11 \\
\hline Maize & 623 & 944 & 939 & 994 & 300 & 718 \\
\hline Cotton $^{3 /}$ & 118 & 149 & 195 & 234 & 159 & 207 \\
\hline Wheat & 2 & 1 & 1 & 0 & 59 & 38 \\
\hline Groundnuts & 38 & 71 & 112 & 156 & 0 & 0 \\
\hline Tea & 3 & 1 & 1 & 0 & 0 & 16 \\
\hline Soybeans & 1 & 3 & 3 & 11 & 7 & 7 \\
\hline Sunflower seeds & 12 & 10 & 7 & 28 & 4 & 9 \\
\hline Rapoko & 5 & 16 & 11 & 22 & 9 & 17 \\
\hline Mhunga & 15 & 25 & 20 & 20 & 4 & 23 \\
\hline Sorghum & 16 & 42 & 29 & 41 & 4 & 9 \\
\hline \multicolumn{7}{|l|}{ Total production } \\
\hline Tobacco (flue cured) & 182 & 169 & 183 & 190 & 173 & 82 \\
\hline Maize & 1,196 & 1,606 & 1,620 & 1,526 & 500 & 929 \\
\hline Cotton $^{3 /}$ & 179 & 197 & 242 & 280 & 190 & 228 \\
\hline Sugarcane & 1,487 & 1,186 & 2,261 & 2,477 & 3,667 & $\ldots$ \\
\hline Wheat & 242 & 287 & 252 & 232 & 213 & 49 \\
\hline Groundnuts & 46 & 80 & 124 & 169 & 59 & 50 \\
\hline Tea & 19 & 15 & 10 & 9 & 22 & 22 \\
\hline Soybeans & 116 & 120 & 135 & 141 & 72 & 70 \\
\hline Coffee & 11 & 8 & 8 & 8 & 9 & 8 \\
\hline Sunflower seeds & 15 & 12 & 10 & 30 & 4 & 10 \\
\hline Rapoko & 6 & 17 & 12 & 23 & 10 & 18 \\
\hline Mhunga & 15 & 25 & 20 & 20 & 4 & 23 \\
\hline Sorghum & 39 & 58 & 46 & 56 & 24 & 32 \\
\hline
\end{tabular}

Sources: Central Statistical Office; Reserve Bank of Zimbabwe; and IMF staff estimates.

1/ Crop season ending in year indicated.

2/ Large- and small-scale commercial farms.

3 / Includes deltapine and delmac cotton.

4/ Communal lands and resettlement areas. 
Table 4. Zimbabwe: Prices of Marketed Agricultural Crops, 1997/98-2002/03 ${ }^{1 /}$ (Unit values in thousands of Zimbabwe dollars per metric ton)

\begin{tabular}{lrrrrrr}
\hline & $1997 / 98$ & $1998 / 99$ & $1999 / 2000$ & $2000 / 01$ & $2001 / 02$ & $2002 / 03$ \\
& & & & & & \\
\hline & 1.2 & 2.4 & 4.2 & 5.4 & 15.0 & 31.9 \\
Maize & 7.1 & 8.7 & 16.6 & 15.9 & 31.1 & 107.4 \\
Cotton & 3.1 & 3.7 & 5.5 & 7.4 & 25.0 & 70.0 \\
Wheat & 26.6 & 34.8 & 66.2 & 83.8 & 174.7 & 361.3 \\
Tobacco; flue cured & 20.3 & 24.8 & 50.7 & 36.9 & 92.0 & 205.8 \\
Tobacco; burley & 2.9 & 5.0 & 6.5 & 9.4 & 17.0 & 70.0 \\
Soybeans & 2.2 & 1.0 & 3.0 & 3.1 & 5.5 & 28.0 \\
Sorghum & 4.0 & 4.0 & 7.0 & 9.6 & 22.0 & 45.0 \\
Groundnuts (unshelled) & 13.8 & 60.0 & 80.0 & 80.0 & 80.0 & 350.0 \\
Coffee & 1.5 & 2.3 & 6.0 & 5.6 & 14.0 & 40.0 \\
Sunflower seeds & & & & & & \\
& & & & & & \\
\end{tabular}

Source: Central Statistical Office.

1/ Marketing years run from April 1 to March 31. 
Table 5. Zimbabwe: Area Under Cultivation for Major Crops, 1998-2003 ${ }^{1 /}$ (In hectares)

\begin{tabular}{lrrrrrr}
\hline & 1998 & 1999 & 2000 & 2001 & $\begin{array}{r}2002 \\
\text { Est. }\end{array}$ & $\begin{array}{r}2003 \\
\text { Est. }\end{array}$ \\
& & & & & & \\
Maize & $1,181,207$ & $1,477,990$ & $1,373,117$ & $1,239,988$ & $1,317,800$ & $1,352,368$ \\
Cotton & 236,287 & 310,534 & 282,469 & 384,574 & 398,600 & 209,127 \\
Tobacco & 79,295 & 78,713 & 76,200 & 66,698 & 71,880 & 47,213 \\
$\quad$ Flue cured & 73,534 & 72,654 & 70,306 & 61,189 & 68,230 & 45,712 \\
$\quad$ Burley & 5,761 & 6,059 & 5,894 & 5,509 & 3,650 & 1,501 \\
Soybeans & 60,290 & 52,931 & 60,650 & 64,009 & 54,500 & 25,390 \\
Sorghum & 126,039 & 143,912 & 116,248 & 110,138 & 82,700 & 128,530 \\
Groundnuts (unshelled) & 178,555 & 132,117 & 175,773 & 275,036 & 259,000 & 105,052 \\
Coffee $^{2 /}$ & 6,741 & 5,912 & 5,215 & 5,516 & 3,792 & $\ldots$ \\
Sugarcane $^{2 /}$ & 37,360 & 39,459 & 39,224 & 39,680 & 37,414 & $\ldots$ \\
Wheat $^{2 /}$ & 41,943 & 52,592 & 47,796 & 42,117 & 32,900 & 16,400 \\
\hline
\end{tabular}

Sources: Central Statistical Office; and Zimbabwe Grain Producers' Association.

1/ Crop season ending in year indicated.

2/ Large-scale commercial farms only. 
Table 6. Zimbabwe: Volume and Value of Livestock Slaughtering and Milk Production, 1998-2003 ${ }^{1 /}$

\begin{tabular}{|c|c|c|c|c|c|c|}
\hline & 1998 & 1999 & 2000 & 2001 & 2002 & 2003 \\
\hline & \multicolumn{6}{|c|}{ (Volume in thousands) } \\
\hline Cattle slaughtering & 369 & 347 & 382 & 347 & 329 & 208 \\
\hline Sheep slaughtering & 22 & 24 & 20 & 21 & 32 & 13 \\
\hline Pig slaughtering & 224 & 199 & 219 & 201 & 177 & 114 \\
\hline \multirow[t]{2}{*}{ Goat slaughtering } & 36 & 25 & 21 & 10 & 14 & 10 \\
\hline & \multicolumn{6}{|c|}{ (Whole-milk tons) } \\
\hline \multirow[t]{2}{*}{ Milk production } & 165,803 & 158,170 & 144,973 & 136,318 & 116,589 & 81,913 \\
\hline & \multicolumn{6}{|c|}{ (Value in millions of Zimbabwe dollars) } \\
\hline Total slaughtering & 2,190 & 4,195 & 6,533 & 7,446 & 13,129 & 63,302 \\
\hline Cattle & 1,901 & 3,714 & 5,835 & 6,204 & 10,035 & 48,419 \\
\hline Sheep & 8 & 11 & 29 & 38 & 102 & 29 \\
\hline Pigs & 274 & 463 & 653 & 1,193 & 2,966 & 14,839 \\
\hline Goats & 7 & 7 & 16 & 11 & 26 & 15 \\
\hline Milk production & 620 & 1,248 & 1,852 & 2,905 & 6,902 & 9,029 \\
\hline
\end{tabular}

Source: Central Statistical Office.

1/ Figures for slaughtering do not include the 3 rd \& 4th quarter slaughterings for butchers $\&$ grading centres. 
Table 7. Zimbabwe: Livestock in Communal and Commercial Farming Areas, 1998-2002 (In thousands)

\begin{tabular}{lrrrrr}
\hline & 1998 & 1999 & 2000 & 2001 & 2002 \\
& & & & & \\
\hline Cattle & 4,953 & 5,316 & 5,226 & 5,541 & 4,817 \\
$\quad$ Communal lands & 3,588 & 3,975 & 3,982 & 4,398 & 4,055 \\
$\quad$ Commercial farms & 1,365 & 1,341 & 1,244 & 1,143 & 762 \\
$\quad$ Beef cattle & 1,275 & 1,259 & 1,169 & 1,071 & 710 \\
$\quad$ Dairy cattle & 90 & 82 & 75 & 72 & 52 \\
& & & & & \\
Sheep & 343 & 310 & 293 & 546 & 532 \\
Communal lands & 274 & 250 & 238 & 493 & 498 \\
Commercial farms & 69 & 60 & 55 & 53 & 34 \\
& 4,645 & 4,363 & 3,848 & 3,426 & 3,170 \\
Goats & 4,616 & 4,339 & 3,828 & 3,407 & 3,156 \\
$\quad$ Communal lands & 29 & 24 & 20 & 19 & 14 \\
Commercial farms & & & & & \\
& 311 & 244 & 250 & 277 & 243 \\
Pigs & 197 & 140 & 160 & 161 & 145 \\
$\quad$ Communal lands & 114 & 104 & 90 & 116 & 98 \\
Commercial farms & & & & & \\
\hline
\end{tabular}

Sources: Central Statistical Office; and Veterinary Services. 
Table 8. Zimbabwe: Volume of Manufacturing Output, 1998-2003

\begin{tabular}{|c|c|c|c|c|c|c|}
\hline & 1998 & 1999 & 2000 & 2001 & 2002 & 2003 \\
\hline & \multicolumn{6}{|c|}{ (Volume indices: period averages, $1990=100$ ) } \\
\hline Foodstuffs and stock feeds & 83.7 & 84.2 & 73.4 & 72.4 & 65.2 & 48.5 \\
\hline Drinks and tobacco & 111.1 & 96.2 & 114.0 & 86.7 & 61.8 & 62.0 \\
\hline Textiles and cotton ginning & 79.1 & 87.3 & 72.8 & 68.9 & 49.4 & 32.0 \\
\hline Clothing and footwear & 114.6 & 125.7 & 120.8 & 123.0 & 108.4 & 104.4 \\
\hline Wood and furniture & 342.8 & 285.0 & 231.1 & 220.0 & 205.8 & 162.9 \\
\hline Paper, printing, and publishing & 102.7 & 94.1 & 74.7 & 69.5 & 69.5 & 61.2 \\
\hline Chemical and petroleum products & 120.3 & 107.4 & 75.9 & 75.2 & 79.1 & 68.0 \\
\hline Non-metallic mineral products & 125.7 & 115.4 & 112.1 & 120.5 & 85.5 & 60.0 \\
\hline Metals and metal products & 77.5 & 82.8 & 82.9 & 73.5 & 65.8 & 65.4 \\
\hline Transport equipment & 101.1 & 69.2 & 52.0 & 43.7 & 41.2 & 38.6 \\
\hline Other manufacturing & 69.2 & 64.1 & 48.6 & 45.0 & 52.1 & 33.4 \\
\hline \multirow[t]{2}{*}{ Overall index } & 106.6 & 99.9 & 93.3 & 84.9 & 72.6 & 64.0 \\
\hline & \multicolumn{6}{|c|}{ (Percent change) } \\
\hline Foodstuffs and stock feeds & -1.3 & 0.6 & -12.8 & -1.3 & -9.9 & -25.6 \\
\hline Drinks and tobacco & 13.0 & -13.4 & 18.5 & -24.0 & -28.7 & 0.3 \\
\hline Textiles and cotton ginning & 7.0 & 10.4 & -16.6 & -5.4 & -28.3 & -35.2 \\
\hline Clothing and footwear & 7.9 & 9.6 & -3.9 & 1.8 & -11.9 & -3.7 \\
\hline Wood and furniture & -2.0 & -16.9 & -18.9 & -4.8 & -6.5 & -20.8 \\
\hline Paper, printing, and publishing & -4.4 & -8.4 & -20.7 & -6.9 & 0.0 & -11.9 \\
\hline Chemical and petroleum products & -5.0 & -10.7 & -29.3 & -1.0 & 5.2 & -14.0 \\
\hline Non-metallic mineral products & 10.1 & -8.2 & -2.8 & 7.4 & -29.0 & -29.8 \\
\hline Metals and metal products & -16.4 & 6.9 & 0.1 & -11.3 & -10.5 & -0.6 \\
\hline Transport equipment & -27.6 & -31.5 & -24.9 & -15.9 & -5.7 & -6.3 \\
\hline Other manufacturing & -14.4 & -7.3 & -24.2 & -7.4 & 15.8 & -35.9 \\
\hline Overall index & -1.3 & -6.3 & -6.6 & -9.0 & -14.5 & -11.8 \\
\hline
\end{tabular}

Source : Central Statistical Office. 
Table 9. Zimbabwe: Mineral Production,1998-2003

\begin{tabular}{|c|c|c|c|c|c|c|}
\hline & 1998 & 1999 & 2000 & 2001 & 2002 & $\begin{array}{r}2003 \\
\text { Jan.-Jul. }\end{array}$ \\
\hline & \multicolumn{6}{|c|}{ (In thousands of metric tonnes, unless otherwise indicated) } \\
\hline \multicolumn{7}{|l|}{ Volume of production } \\
\hline Gold (thousands of fine ounces) & 809 & 872 & 708 & 560 & 431 & 218 \\
\hline Platinum (kilograms) & 2,685 & 390 & 504 & 435 & 2,011 & 1,719 \\
\hline Nickel & 10,173 & 9,593 & 5,967 & 3,908 & 6,313 & 3,112 \\
\hline Coal & 5,467 & 4,977 & 3,986 & 4,511 & 3,938 & 1,768 \\
\hline Asbestos & 123 & 88 & 152 & 119 & 168 & 94 \\
\hline Copper & 2.0 & 5.0 & 0.6 & 0.5 & 1.3 & 0.9 \\
\hline Chrome ore & 605 & 653 & 668 & 723 & 672 & 334 \\
\hline Iron ore & 372 & 599 & 438 & 362 & 272 & 256 \\
\hline Silver (thousands of fine ounces) & 531 & 173 & 122 & 108 & 92 & 50 \\
\hline Cobalt (metric tons) & 94 & 131 & 79 & 68 & 105 & 32 \\
\hline Black granite & 226 & 124 & 510 & 379 & 197 & 114 \\
\hline & \multicolumn{6}{|c|}{$($ Index, $1990=100)$} \\
\hline \multicolumn{7}{|l|}{ Unit value index ${ }^{1 /}$} \\
\hline Gold & 742 & 1,139 & 1,295 & 1,541 & 1,759 & 60,960 \\
\hline Platinum & 1,114 & 706 & 2,787 & 18,094 & 22,723 & 41,518 \\
\hline Nickel & 501 & 1,007 & 1,769 & 2,841 & 10,043 & 45,445 \\
\hline Coal & 667 & 1,105 & 2,072 & 3,121 & 6,131 & 12,856 \\
\hline Asbestos & 1,169 & 1,849 & 2,024 & 2,253 & 2,257 & 30658 \\
\hline Copper & 668 & 954 & 711 & 1,196 & 6,241 & 16,254 \\
\hline Chrome ore & 421 & 707 & 1,101 & 2,001 & 5,789 & 23,433 \\
\hline Iron ore & 446 & 693 & 1,018 & 2,818 & 4,425 & 6,005 \\
\hline Silver & 452 & 1,667 & 1,825 & 1,970 & 2,073 & 23,201 \\
\hline Cobalt & 1,725 & 1,791 & 1,587 & 5,794 & 25,767 & 6,204 \\
\hline \multicolumn{7}{|l|}{ Memorandum item: } \\
\hline $\begin{array}{l}\text { Exchange rate (Zimbabwe dollars per } \\
\text { U.S. dollar; period average) }\end{array}$ & 23.68 & 38.30 & 44.42 & 55.05 & 55.04 & 549.37 \\
\hline
\end{tabular}

Sources: Ministry of Finance; Central Statistical Office; and IMF, International Financial Statistics .

1/ Unit value indices are estimates. 
Table 10. Zimbabwe: Construction and Retail Trade, 1998-2003

\begin{tabular}{|c|c|c|c|c|c|c|}
\hline & 1998 & 1999 & 2000 & 2001 & 2002 & $\begin{array}{r}2003 \\
\text { Jan.-Sep. }\end{array}$ \\
\hline & \multicolumn{6}{|c|}{ (In millions of Zimbabwe dollars, unless otherwise indicated) } \\
\hline \multicolumn{7}{|l|}{ Construction indicators } \\
\hline Total building plans approved ${ }^{1 /}$ & 1,910 & 2,908 & 3,619 & 5,119 & 8,592 & 1,053 \\
\hline Municipal building plans approved & 4,208 & 4,332 & 4,539 & $\ldots$ & $\ldots$ & $\ldots$ \\
\hline Low-cost houses and flats (in units) & 2,503 & 1,899 & 1,250 & 588 & 420 & 35 \\
\hline High-cost houses and flats (in units) & 1,705 & 2,433 & 3,289 & $\ldots$ & $\ldots$ & $\ldots$ \\
\hline \multicolumn{7}{|l|}{ Work done by private and public contractors } \\
\hline Public & 752 & 325 & 139 & $\ldots$ & $\ldots$ & $\ldots$ \\
\hline Private & 1,741 & 1,755 & $\ldots$ & $\ldots$ & $\ldots$ & $\ldots$ \\
\hline Of which: public sector & 284 & 360 & $\ldots$ & $\ldots$ & $\ldots$ & $\ldots$ \\
\hline Retail trade value index, monthly average $1980=100^{2 /}$ & 1,378 & $\ldots$ & $\ldots$ & $\ldots$ & $\ldots$ & $\ldots$ \\
\hline (Percent change) & 11 & $\ldots$ & $\ldots$ & $\ldots$ & $\ldots$ & $\ldots$ \\
\hline
\end{tabular}

Source: Central Statistical Office

1/ Includes additions and alterations.

2/ 1998 index through December. 
Table 11. Zimbabwe: Electrical Energy Produced and Distributed, 1998-2003

(In millions of kilowatt-hours)

\begin{tabular}{|c|c|c|c|c|c|c|}
\hline & 1998 & 1999 & 2000 & 2001 & 2002 & $\begin{array}{r}2003 \\
\text { Jan.-Oct. }\end{array}$ \\
\hline Total power distributed & 11,620 & 12,408 & 12,206 & 12,206 & 12,583 & 10,242 \\
\hline From central grid ${ }^{1 /}$ & 11,515 & 12,290 & 12,102 & 12,164 & 9,548 & 10,242 \\
\hline Noninterconnected thermal station & 102 & 114 & 104 & 42 & 0 & 0 \\
\hline Other (net) $)^{2 /}$ & 3 & 4 & 3 & 0 & 0 & 0 \\
\hline Power generated domestically & 6,677 & 7,208 & 7,099 & 7,968 & 7,562 & 9,173 \\
\hline From South Kariba ${ }^{3 /}$ & 1,925 & 2,950 & 3,260 & 2,998 & 3,824 & 4,410 \\
\hline Thermal stations & 4,650 & 4,140 & 3,735 & 4,928 & 3,738 & 4,763 \\
\hline Noninterconnected thermal station & 102 & 118 & 104 & 42 & 0 & 0 \\
\hline Net imports & 4,936 & 5,204 & 5,106 & 4,185 & 3,046 & 2,961 \\
\hline
\end{tabular}

Source: Central Statistical Office.

1/ Drawings from the grid of the Central African Power Corporation.

2/ Net imports from noninterconnected sources.

3/ Power generated from South Kariba is Zimbabwe's share but it is fed into the Central African Power Corporation Grid. 
Table 12. Zimbabwe: Petroleum Products, 1998-2004

\begin{tabular}{|c|c|c|c|c|c|c|c|}
\hline & 1998 & 1999 & 2000 & 2001 & 2002 & 2003 & $\begin{array}{l}2004 \\
\text { Mar. }\end{array}$ \\
\hline \multicolumn{8}{|c|}{ Retail price (Zimbabwe cents per liter; end of period) ${ }^{1 /}$} \\
\hline Premium petrol & 967 & 1,868 & 4,380 & 7,447 & 7,447 & 175,000 & 175,000 \\
\hline Diesel & 810 & 1,681 & 3,960 & 6,639 & 6,639 & 165,000 & 165,000 \\
\hline Aviation gas & 1,214 & 1,865 & 2,026 & 2,026 & 5,737 & 247,800 & 247,800 \\
\hline Jet fuel (A1) & 792 & 1,669 & 3,120 & 5,703 & 5,703 & 247,800 & 247,800 \\
\hline Liquefied petroleum gas (LPG) & 1,400 & 2,405 & 2,566 & 2,556 & 10,500 & 10,500 & 10,500 \\
\hline \multicolumn{8}{|c|}{ Tax (Zimbabwe cents per liter; end of period) } \\
\hline Premium petrol & 140 & 346 & 912 & 730 & 730 & 730 & 5,850 \\
\hline Diesel & 50 & 188 & 385 & 385 & 385 & 385 & 5,850 \\
\hline Aviation gas & 50 & 50 & 50 & 0 & 0 & 0 & 0 \\
\hline Jet fuel (A1) & 50 & 304 & 0 & 0 & 0 & 0 & 0 \\
\hline LPG & 100 & 100 & 100 & 100 & 54 & 54 & 5,850 \\
\hline \multicolumn{8}{|c|}{ Quantity imported (thousands of liters) $^{1 /}$} \\
\hline Premium petrol & 530,252 & 709,469 & 381,234 & 473,310 & 389,494 & 15,469 & 6,000 \\
\hline Diesel & 956,480 & $1,215,446$ & 632,828 & 525,263 & 681,921 & 32,412 & 28,779 \\
\hline Aviation gas & 3,433 & 4,513 & 0 & 0 & 300 & 90 & 0 \\
\hline Jet fuel (A1) & 311,289 & 305,840 & 140,917 & 120,723 & 79,013 & 426 & 6,362 \\
\hline LPG & 7,071 & 7,012 & 755 & 1,073 & 1,317 & 0 & 0 \\
\hline
\end{tabular}

Sources: National Oil Company of Zimbabwe; and Zimbabwean news sources.

1/ Data for 2003 and 2004 is for government imports only. Data on retail prices and quantity imported by the private sector is not available. 
Table 13. Zimbabwe: Consumer Price Index, 1998-2004

\begin{tabular}{|c|c|c|c|c|c|c|c|c|}
\hline & Weight & 1998 & 1999 & 2000 & 2001 & 2002 & 2003 & $\begin{array}{r}2004 \\
\text { Apr. }\end{array}$ \\
\hline & & \multicolumn{7}{|c|}{$($ Index, 1995=100) } \\
\hline Food & 33.6 & 250 & 392 & 559 & 1,164 & 3,725 & 27,399 & 37,606 \\
\hline Nonfood index & 66.4 & 376 & 592 & 1,118 & 2,467 & 6,352 & 22,858 & 30,084 \\
\hline Beverages and tobacco & 16.0 & 236 & 396 & 617 & 1,215 & 5,551 & 38,246 & 44,630 \\
\hline Clothing and footwear & 6.9 & 164 & 287 & 437 & 1,129 & 3,652 & 22,833 & 31,130 \\
\hline Rent rates and power & 17.3 & 188 & 255 & 410 & 744 & 1,253 & 5,704 & 13,855 \\
\hline Furniture and household goods & 7.5 & 218 & 339 & 505 & 1,532 & 3,779 & 22,858 & 27,359 \\
\hline Medical care & 1.7 & 181 & 351 & 539 & 1,083 & 2,884 & 19,298 & 24,813 \\
\hline Transport and communication & 6.6 & 255 & 419 & 899 & 1,922 & 3,460 & 41,889 & 51,258 \\
\hline Recreation and entertainment & 1.2 & 222 & 386 & 682 & 2,178 & 5,280 & 29,361 & 43,255 \\
\hline Education & 4.5 & 207 & 284 & 410 & 850 & 1,877 & 9,870 & 17,609 \\
\hline Miscellaneous & 4.7 & 233 & 328 & 474 & 1,247 & 3,180 & 17,536 & 22,116 \\
\hline \multirow[t]{2}{*}{ Total index } & 100.0 & 226 & 355 & 550 & 1,167 & 3,490 & 24,384 & 32,611 \\
\hline & & \multicolumn{7}{|c|}{$(\text { Year-on-year percent change })^{1 /}$} \\
\hline Food & & 62.9 & 56.8 & 42.9 & 108.0 & 220.1 & 635.6 & 563.5 \\
\hline Beverages and tobacco & & 66.7 & 65.3 & 55.5 & 97.0 & 357.1 & 589.0 & 457.4 \\
\hline Clothing and footwear & & 27.9 & 75.2 & 52.4 & 158.5 & 223.5 & 525.2 & 448.9 \\
\hline Rent rates and power & & 11.2 & 35.8 & 60.4 & 81.7 & 68.4 & 355.1 & 463.2 \\
\hline Furniture and household goods & & 52.1 & 55.5 & 48.9 & 203.6 & 146.7 & 504.9 & 416.5 \\
\hline Medical care & & 15.6 & 94.5 & 53.2 & 101.1 & 166.3 & 569.1 & 522.8 \\
\hline Transport and communication & & 62.3 & 64.4 & 114.8 & 113.7 & 80.0 & $1,110.6$ & 626.9 \\
\hline Recreation and entertainment & & 46.4 & 73.5 & 76.7 & 219.6 & 142.4 & 456.1 & 395.5 \\
\hline Education & & 22.4 & 36.7 & 44.7 & 107.1 & 120.9 & 425.9 & 514.3 \\
\hline Miscellaneous & & 53.1 & 40.7 & 44.5 & 163.1 & 155.0 & 451.4 & 406.8 \\
\hline Total index & & 46.7 & 56.9 & 55.2 & 112.1 & 198.9 & 598.7 & 505.0 \\
\hline
\end{tabular}

Source: Central Statistical Office.

1/ December over December. 


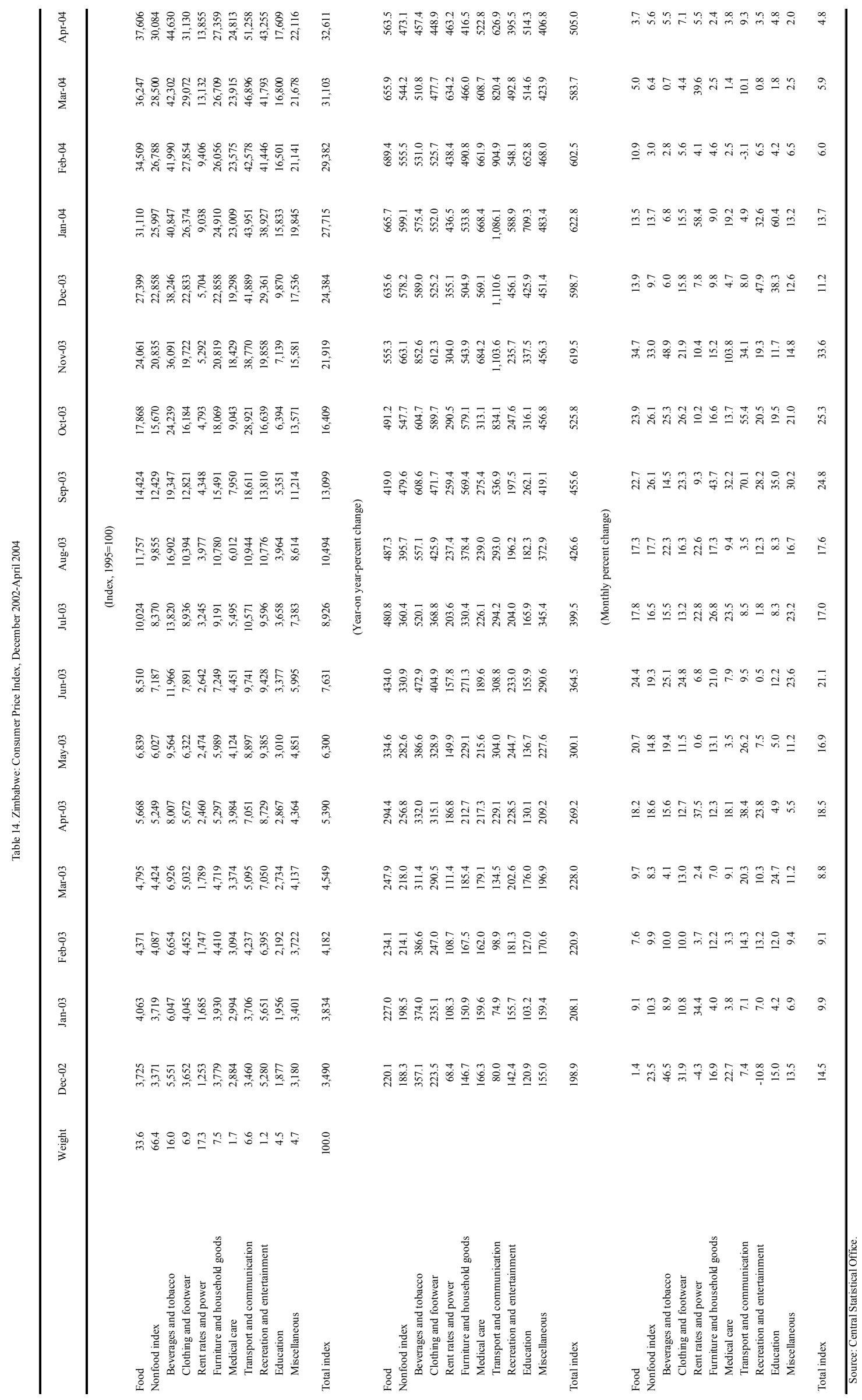


Table 15. Zimbabwe: Employment and Employment Earnings, 1998-2002

\begin{tabular}{|c|c|c|c|c|c|}
\hline & 1998 & 1999 & 2000 & 2001 & 2002 \\
\hline Employment & \multicolumn{5}{|c|}{ (Thousands of employees; period average) } \\
\hline Agriculture, forestry and fishing & 345 & 338 & 325 & 290 & 221 \\
\hline Mining and quarrying & 61 & 60 & 45 & 43 & 43 \\
\hline Manufacturing & 208 & 201 & 181 & 179 & 171 \\
\hline Electricity and water & 16 & 17 & 11 & 9 & 10 \\
\hline Construction & 79 & 69 & 54 & 41 & 42 \\
\hline Finance, insurance and real estate & 28 & 31 & 35 & 28 & 28 \\
\hline Distribution, restaurants and hotels & 113 & 115 & 103 & 100 & 105 \\
\hline Transport and communication & 51 & 45 & 44 & 42 & 41 \\
\hline Public administration & 66 & 63 & 59 & 61 & 65 \\
\hline Education & 146 & 135 & 140 & 148 & 149 \\
\hline Health & 28 & 28 & 28 & 32 & 32 \\
\hline Domestic & 102 & 102 & 102 & 102 & 102 \\
\hline Other & 105 & 113 & 107 & 108 & 105 \\
\hline Total & 1,348 & 1,317 & 1,234 & 1,183 & 1,114 \\
\hline Government $^{1 /}$ & 240 & 226 & 227 & 241 & 246 \\
\hline Non-government & 1,108 & 1,091 & 1,007 & 942 & 868 \\
\hline Earnings & \multicolumn{5}{|c|}{ (In millions of Zimbabwe dollars) } \\
\hline Agriculture, forestry and fishing & 2,977 & 4,163 & 5,725 & 10,727 & \\
\hline Mining and quarrying & 2,957 & 4,122 & 4,331 & 7,059 & 16,732 \\
\hline Manufacturing & 9,216 & 12,782 & 18,078 & 30,054 & 58,070 \\
\hline Electricity and water & 2,586 & 3,440 & 4,299 & 6,810 & 10,251 \\
\hline Construction & 2,282 & 2,549 & 4,173 & 4,744 & 7,881 \\
\hline Finance, insurance and real estate & 3,619 & 5,305 & 10,241 & 13,234 & 23,040 \\
\hline Distribution, restaurants and hotels & 4,775 & 7,022 & 9,833 & 15,645 & 29,577 \\
\hline Transport and communication & 3,156 & 4,395 & 7,463 & 10,439 & 17,379 \\
\hline Public administration & 3,924 & 5,728 & 12,682 & 12,954 & 29,070 \\
\hline Education & 10,360 & 14,914 & 30,116 & 39,222 & 74,768 \\
\hline Health & 2,089 & 2,906 & 7,031 & 8,502 & 18,958 \\
\hline Domestic & 191 & 191 & 191 & 191 & 191 \\
\hline Other & 4,043 & 5,921 & 10,205 & 14,044 & 28,132 \\
\hline Total & 52,175 & 73,438 & 124,368 & 173,625 & $\ldots$ \\
\hline
\end{tabular}

Source: Central Statistical Office (CSO).

1/ Public administration, health and education. 
Table 16. Zimbabwe: Central Government Operations, 1999-2003

\begin{tabular}{|c|c|c|c|c|c|}
\hline & 1999 & 2000 & 2001 & 2002 & 2003 \\
\hline & \multicolumn{5}{|c|}{ (In billions of Zimbabwe dollars) } \\
\hline Total revenue & 58.6 & 87.8 & 136.0 & 304.2 & $1,374.7$ \\
\hline Tax revenue & 55.6 & 82.3 & 128.5 & 284.6 & $1,325.8$ \\
\hline Income and profits & 29.7 & 52.2 & 73.8 & 158.3 & 734.7 \\
\hline Customs duties & 8.5 & 8.5 & 17.4 & 27.2 & 92.9 \\
\hline Excise duties & 2.9 & 4.1 & 5.3 & 18.8 & 94.6 \\
\hline Sales tax & 12.3 & 15.7 & 29.3 & 76.2 & 382.3 \\
\hline Other taxes & 2.1 & 1.7 & 2.7 & 4.0 & 21.3 \\
\hline Nontax revenue & 3.0 & 5.6 & 7.4 & 19.6 & 49.0 \\
\hline Total expenditure and net lending & 80.2 & 158.7 & 188.9 & 351.3 & $1,394.6$ \\
\hline Current expenditure & 71.3 & 153.2 & 176.8 & 320.7 & $1,233.2$ \\
\hline Goods and services & 40.6 & 79.8 & 99.9 & 216.0 & 924.3 \\
\hline Salaries and wages & 28.2 & 59.5 & 64.5 & 123.9 & 528.0 \\
\hline Other & 12.5 & 20.3 & 35.4 & 92.1 & 396.4 \\
\hline Interest on debt & 21.6 & 53.0 & 52.8 & 49.5 & 69.2 \\
\hline Foreign & 3.6 & 1.6 & 11.7 & 9.2 & 3.3 \\
\hline Domestic & 18.1 & 51.5 & 41.1 & 40.3 & 65.9 \\
\hline Transfers & 9.0 & 20.4 & 24.1 & 55.2 & 239.7 \\
\hline Capital expenditure & 6.3 & 2.4 & 11.6 & 25.2 & 107.7 \\
\hline Net lending & 2.6 & 3.1 & 0.6 & 5.4 & 53.7 \\
\hline Balance, excluding grants and foreign interest arrears ${ }^{1 /}$ & -21.6 & -70.9 & -53.0 & -47.2 & -19.8 \\
\hline Grants & 2.1 & 3.5 & 3.0 & 0.7 & 6.1 \\
\hline Foreign interest arrears & 0.2 & 2.8 & 11.5 & 8.8 & 3.3 \\
\hline Balance, including grants and foreign interest arrears ${ }^{1 /}$ & -19.3 & -64.6 & -38.5 & -37.7 & -10.5 \\
\hline Financing & 19.3 & 64.6 & 38.5 & 37.7 & 10.5 \\
\hline Foreign financing & -2.3 & 2.7 & 0.7 & -1.5 & 0.9 \\
\hline Borrowing & 6.2 & 7.2 & 1.4 & 0.2 & 0.9 \\
\hline Repayments & -8.5 & -4.5 & -0.7 & -1.7 & 0.0 \\
\hline Domestic financing & 21.6 & 61.9 & 37.8 & 39.2 & 9.6 \\
\hline \multirow[t]{2}{*}{ Of which: proceeds of asset sales } & 0.3 & 0.0 & 6.7 & 0.5 & 0.0 \\
\hline & \multicolumn{5}{|c|}{ (In percent of GDP, unless otherwise indicated) } \\
\hline Total revenue & 26.4 & 26.7 & 24.1 & 25.2 & 23.7 \\
\hline Tax revenue & 25.1 & 25.0 & 22.8 & 23.6 & 22.8 \\
\hline Income and profits & 13.4 & 15.9 & 13.1 & 13.1 & 12.6 \\
\hline Customs duties & 3.8 & 2.6 & 3.1 & 2.3 & 1.6 \\
\hline Other domestic & 7.8 & 6.6 & 6.6 & 8.2 & 8.6 \\
\hline Nontax Revenue & 1.4 & 1.7 & 1.3 & 1.6 & 0.8 \\
\hline Total expenditure & 36.2 & 48.3 & 33.5 & 29.2 & 24.0 \\
\hline Current expenditure & 32.2 & 46.6 & 31.3 & 26.6 & 21.2 \\
\hline Goods and services & 18.3 & 24.3 & 17.7 & 17.9 & 15.9 \\
\hline Salaries and wages & 12.7 & 18.1 & 11.4 & 10.3 & 9.1 \\
\hline Other & 5.6 & 6.2 & 6.3 & 7.6 & 6.8 \\
\hline Interest on debt & 9.8 & 16.1 & 9.4 & 4.1 & 1.2 \\
\hline Foreign & 1.6 & 0.5 & 2.1 & 0.8 & 0.1 \\
\hline Domestic & 8.2 & 15.7 & 7.3 & 3.3 & 1.1 \\
\hline Subsidies & 0.0 & 0.0 & 0.0 & 0.0 & 0.0 \\
\hline Transfers & 4.1 & 6.2 & 4.3 & 4.6 & 4.1 \\
\hline Capital expenditure & 2.9 & 0.7 & 2.1 & 2.1 & 1.9 \\
\hline Net lending & 1.2 & 1.0 & 0.1 & 0.4 & 0.9 \\
\hline Balance, excluding grants ${ }^{1 /}$ & -9.8 & -21.6 & -9.4 & -3.9 & -0.3 \\
\hline \multicolumn{6}{|l|}{ Memorandum item: } \\
\hline GDP at market prices (Z\$ billions) & 222 & 329 & 565 & 1,205 & 5,810 \\
\hline
\end{tabular}

Sources: Zimbabwean authorities; and IMF staff estimates.

1/ On a commitment basis 
Table 17. Zimbabwe: Detailed Central Government Revenue, 1999-2003

(In billions of Zimbabwe dollars)

\begin{tabular}{|c|c|c|c|c|c|}
\hline & 1999 & 2000 & 2001 & 2002 & 2003 \\
\hline Total revenue & 58.6 & 87.8 & 136.0 & 304.2 & $1,374.7$ \\
\hline Tax revenue & 55.6 & 82.3 & 128.5 & 284.6 & $1,325.8$ \\
\hline Income and profits & 29.7 & 52.2 & 73.8 & 158.3 & 734.7 \\
\hline Individuals & 19.6 & 35.9 & 53.1 & 116.4 & 588.8 \\
\hline Companies & 6.8 & 9.6 & 14.9 & 30.0 & 80.6 \\
\hline Domestic dividends and interest & 1.4 & 3.3 & 2.6 & 4.5 & 22.2 \\
\hline Other income taxes & 1.9 & 3.5 & 3.2 & 7.5 & 43.2 \\
\hline Customs duties & 8.5 & 8.5 & 17.4 & 27.2 & 92.9 \\
\hline Oil products & 0.8 & 0.1 & 3.3 & 5.5 & 0.7 \\
\hline Other & 7.7 & 8.5 & 14.1 & 21.7 & 92.1 \\
\hline Excise duties & 2.9 & 4.1 & 5.3 & 18.8 & 94.6 \\
\hline Beer & 1.7 & 2.3 & 2.7 & 12.4 & 65.0 \\
\hline Tobacco & 0.5 & 0.8 & 1.2 & 0.4 & 0.9 \\
\hline Wine and spirits & 0.1 & 0.2 & 0.2 & 3.4 & 20.7 \\
\hline Other beverages & 0.5 & 0.8 & 1.2 & 2.6 & 8.0 \\
\hline Sales tax & 12.3 & 15.7 & 29.3 & 76.2 & 382.3 \\
\hline Other taxes & 2.1 & 1.7 & 2.7 & 4.0 & 21.3 \\
\hline Tobacco levy & 1.2 & 1.0 & 1.1 & 0.9 & 3.5 \\
\hline Other & 1.0 & 0.7 & 1.6 & 3.1 & 17.8 \\
\hline Nontax revenue & 3.0 & 5.6 & 7.4 & 19.6 & 49.0 \\
\hline Investments and property & 0.5 & 1.3 & 1.7 & 1.2 & 1.8 \\
\hline Reserve Bank remittances & 0.1 & 0.1 & 0.2 & 0.4 & 0.0 \\
\hline Local government interest & 0.0 & 0.0 & 0.2 & 0.1 & 0.0 \\
\hline Parastatal interest, dividends, and oth & 0.4 & 1.1 & 1.4 & 0.7 & 1.8 \\
\hline Fees & 0.3 & 0.5 & 0.7 & 1.1 & 2.5 \\
\hline Other & 2.2 & 3.8 & 5.0 & 17.3 & 44.7 \\
\hline
\end{tabular}

Sources: Zimbabwean authorities; and IMF staff estimates. 
Table 18. Zimbabwe: Detailed Central Government Expenditure and Net Lending, 1999-2003 (In billions of Zimbabwe dollars)

\begin{tabular}{|c|c|c|c|c|c|}
\hline & 1999 & 2000 & 2001 & 2002 & 2003 \\
\hline Total expenditure and net lending & 80.2 & 158.7 & 188.9 & 351.3 & $1,394.6$ \\
\hline Current expenditure & 71.3 & 153.2 & 176.8 & 320.7 & $1,233.2$ \\
\hline Current expenditure on goods and servic & 40.6 & 79.8 & 99.9 & 216.0 & 924.3 \\
\hline Wages and salaries & 28.2 & 59.5 & 64.5 & 123.9 & 528.0 \\
\hline Other goods and services & 12.5 & 20.3 & 35.4 & 92.1 & 396.4 \\
\hline Subsistence and transport & 1.3 & 1.9 & 2.6 & 6.4 & 34.1 \\
\hline Incidental expenses & 2.3 & 4.6 & 7.2 & 20.8 & 43.7 \\
\hline Maintenance of capital works & 0.9 & 2.2 & 2.2 & 3.9 & 18.3 \\
\hline Other & 8.1 & 11.6 & 23.5 & 61.1 & 300.3 \\
\hline Interest payments & 21.6 & 53.0 & 52.8 & 49.5 & 69.2 \\
\hline Foreign (commitment) & 3.6 & 1.6 & 11.7 & 9.2 & 3.3 \\
\hline Domestic & 18.1 & 51.5 & 41.1 & 40.3 & 65.9 \\
\hline Subsidies and transfers & 9.0 & 20.4 & 24.1 & 55.2 & 239.7 \\
\hline Of which: pensions & 4.0 & 8.0 & 11.1 & 22.6 & 77.2 \\
\hline Capital expenditure and net lending & 8.9 & 5.5 & 12.2 & 30.6 & 161.4 \\
\hline Capital expenditure & 6.3 & 2.4 & 11.6 & 25.2 & 107.7 \\
\hline Net lending & 2.6 & 3.1 & 0.6 & 5.4 & 53.7 \\
\hline Long-term loans (net) & 2.3 & 2.8 & 0.6 & 5.1 & 2.3 \\
\hline Gross lending & 2.5 & 2.9 & 0.9 & 5.2 & 2.3 \\
\hline Recoveries & -0.2 & -0.1 & -0.4 & -0.1 & 0.0 \\
\hline Investments & 0.0 & 0.5 & 0.0 & 0.3 & 0.0 \\
\hline Short-term loans (net) & 0.2 & -0.1 & 0.0 & -0.1 & 51.4 \\
\hline
\end{tabular}

Sources: Zimbabwean authorities; and IMF staff estimates. 
Table 19. Zimbabwe: Expenditure and Repayments by Ministries, 1999-2003

(In billions of Zimbabwe dollars)

\begin{tabular}{|c|c|c|c|c|c|}
\hline & 1999 & 2000 & 2001 & 2002 & 2003 \\
\hline Total & 79.8 & 175.1 & 169.9 & 343.7 & $1,284.4$ \\
\hline Constitutional and statutory appropriations & 26.1 & 74.2 & 52.1 & 66.3 & 146.0 \\
\hline President and Cabinet & 0.0 & 0.0 & 0.0 & 0.0 & 0.0 \\
\hline Parliament of Zimbabwe & 0.0 & 0.0 & 0.0 & 0.0 & 0.0 \\
\hline Public Service, Labour and Social Welfare & 4.0 & 8.0 & 11.1 & 22.6 & 77.2 \\
\hline Finance and Economic Development & 22.1 & 66.2 & 40.9 & 43.6 & 68.3 \\
\hline Of which: repayments of loans & -7.5 & -10.0 & -34.2 & -2.6 & -2.4 \\
\hline Audit & 0.0 & 0.0 & 0.0 & 0.0 & 0.0 \\
\hline Local Government, Public Works and National Housing & 0.0 & 0.0 & 0.0 & 0.0 & 0.0 \\
\hline Justice, Legal and Parliamentary Affairs & 0.0 & 0.0 & 0.0 & 0.1 & 0.5 \\
\hline Transport and Communications & 0.0 & 0.0 & 0.0 & 0.0 & 0.0 \\
\hline Vote appropriations & 53.7 & 100.8 & 117.8 & 277.4 & $1,138.4$ \\
\hline President and Cabinet & 1.7 & 2.6 & 3.0 & 6.9 & 36.3 \\
\hline Parliament of Zimbabwe & 0.1 & 0.2 & 0.3 & 0.5 & 4.7 \\
\hline Public Service, Labour and Social Welfare & 1.0 & 2.4 & 5.5 & 15.4 & 67.9 \\
\hline Defense & 10.1 & 15.4 & 15.8 & 37.3 & 148.8 \\
\hline Finance and Economic Development & 0.9 & 3.6 & 4.4 & 15.3 & 11.0 \\
\hline Audit & 0.0 & 0.1 & 0.1 & 0.3 & 1.1 \\
\hline Industry and International Trade & 0.3 & 0.3 & 0.4 & 5.1 & 13.3 \\
\hline Lands, Agriculture and Rural Resettlement & 1.6 & 2.8 & 5.1 & 29.0 & 125.8 \\
\hline Mines and Energy & 0.3 & 0.4 & 0.6 & 0.7 & 3.4 \\
\hline Foreign Affairs & 1.4 & 2.6 & 1.4 & 2.1 & 9.2 \\
\hline Local Government, Public Works and National Housing & 1.2 & 2.0 & 2.6 & 10.1 & 26.8 \\
\hline Health and Child Welfare & 4.6 & 9.3 & 13.6 & 34.4 & 130.4 \\
\hline Education, Sport and Culture & 13.5 & 25.6 & 36.0 & 58.0 & 270.1 \\
\hline Higher Education and Technology & 3.4 & 6.7 & 7.2 & 14.2 & 65.4 \\
\hline Home Affairs & 3.5 & 7.1 & 8.9 & 21.9 & 98.3 \\
\hline Justice, Legal and Parlimentary Affairs & 1.0 & 2.0 & 3.2 & 8.7 & 36.5 \\
\hline Transport and Communications & 2.0 & 1.8 & 2.3 & 5.8 & 34.5 \\
\hline Vote of Credit & 5.4 & 13.7 & 4.0 & 2.9 & 5.0 \\
\hline Rural Resources and Water Development & 1.3 & 1.8 & 2.1 & 5.5 & 22.9 \\
\hline Environment and Tourism & 0.0 & 0.0 & 0.4 & 1.0 & 3.4 \\
\hline Youth Development, Gender, and Employment Creation & 0.0 & 0.5 & 0.7 & 2.2 & 8.6 \\
\hline Other & 0.3 & 0.1 & 0.0 & 0.0 & 15.0 \\
\hline
\end{tabular}

Sources: Zimbabwean authorities; and IMF staff estimates. 
Table 20. Zimbabwe: Civil Service Employment Budgeted Posts, 1999-2003

\begin{tabular}{|c|c|c|c|c|c|}
\hline & 1999 & 2000 & 2001 & 2002 & 2003 \\
\hline Total $^{1 /}$ & 163,772 & 161,932 & 160,956 & 167,779 & 173,708 \\
\hline Education, Sport and Culture & 101,894 & 103,881 & 103,806 & 103,806 & 108,478 \\
\hline Higher Education and Technology & 3,681 & 2,901 & 3,112 & 3,112 & 3,112 \\
\hline Health and Child Welfare & 25,171 & 25,171 & 25,430 & 25,430 & 25,430 \\
\hline Lands, Agriculture and Rural Resettlement & 9,865 & 8,767 & 8,965 & 8,970 & 15,530 \\
\hline Transport and Communications & 2,321 & 803 & 905 & 892 & 905 \\
\hline Other & 20,840 & 20,409 & 18,738 & 25,569 & 20,253 \\
\hline \multicolumn{6}{|l|}{ Memorandum items: } \\
\hline GDP (in millions of Zimbabwe dollars) & 228,434 & 328,658 & 564,640 & $1,205,120$ & $5,810,253$ \\
\hline Wage bill (millions of Zimbabwe dollars) & 28,175 & 59,468 & 64,480 & 123,930 & 527,978 \\
\hline Wage bill (in percent of GDP) $)^{2 /}$ & 12.3 & 18.1 & 11.4 & 10.3 & 9.1 \\
\hline
\end{tabular}

Sources: Zimbabwean authorities; and IMF staff estimates.

1/ The number of authorized posts at the beginning of each time period indicated, excluding uniformed services.

2/ Including wages for Defense, Zimbabwe Republic Police, and Prison Service. 
Table 21. Zimbabwe: Central Government Debt and Debt-Service Payments, 1999-2003

\begin{tabular}{|c|c|c|c|c|c|}
\hline & 1999 & 2000 & 2001 & 2002 & 2003 \\
\hline & \multicolumn{5}{|c|}{ (In billions of Zimbabwe dollars) } \\
\hline Total government debt & 139.2 & 244.7 & 270.4 & 338.2 & 448.1 \\
\hline Domestic debt ${ }^{1 /}$ & 62.8 & 121.0 & 137.6 & 202.3 & 315.4 \\
\hline Stocks & 8.3 & 9.3 & 15.2 & 13.7 & 13.5 \\
\hline Bonds & 0.1 & 0.4 & 0.1 & 0.1 & 0.1 \\
\hline Loans & 0.0 & 0.0 & 0.0 & 0.0 & 0.0 \\
\hline Public enterprise debt assumed & 0.4 & 0.0 & 0.0 & 0.0 & 0.0 \\
\hline Floating debt & 53.9 & 111.3 & 122.3 & 188.5 & 301.8 \\
\hline Treasury bills & 53.9 & 111.2 & 122.3 & 188.5 & 301.8 \\
\hline Overdrafts and other & 0.0 & 0.1 & 0.0 & 0.0 & 0.0 \\
\hline External debt ${ }^{2 /}$ & 76.5 & 123.7 & 132.8 & 135.9 & 132.7 \\
\hline Stocks & 0.0 & 0.0 & 0.0 & 0.0 & 0.0 \\
\hline Bonds & 0.2 & 0.1 & 0.1 & 0.1 & 0.1 \\
\hline Loans & 76.3 & 123.6 & 132.7 & 135.8 & 132.6 \\
\hline \multicolumn{6}{|l|}{ Debt service } \\
\hline Debt service due (commitments) & 30.1 & 57.5 & 42.8 & 43.1 & 66.2 \\
\hline Interest & 21.5 & 52.5 & 41.3 & 40.7 & 65.9 \\
\hline Domestic & 18.1 & 51.0 & 41.1 & 40.3 & 65.9 \\
\hline Foreign & 3.4 & 1.5 & 0.2 & 0.4 & 0.0 \\
\hline Repayments of principal ${ }^{3 /}$ & 8.6 & 5.0 & 1.4 & 2.4 & 0.3 \\
\hline Domestic & 0.8 & 0.7 & 0.8 & 0.7 & 0.2 \\
\hline Foreign & 7.8 & 4.3 & 0.6 & 1.7 & 0.0 \\
\hline Debt-service arrears & 0.0 & 1.3 & 25.4 & 40.2 & 53.4 \\
\hline Interest & 0.0 & 0.4 & 6.3 & 9.7 & 14.0 \\
\hline Domestic & 0.0 & 0.0 & 0.0 & 0.0 & 0.0 \\
\hline Foreign & 0.0 & 0.4 & 6.3 & 9.7 & 14.0 \\
\hline Repayments of principal ${ }^{3 /}$ & 0.0 & 0.8 & 19.1 & 30.5 & 39.4 \\
\hline Domestic & 0.0 & 0.0 & 0.0 & 0.0 & 0.0 \\
\hline Foreign & 0.0 & 0.8 & 19.1 & 30.5 & 39.4 \\
\hline & \multicolumn{5}{|c|}{ (In percent of GDP, unless otherwise indicated) } \\
\hline Total government debt & 60.9 & 74.5 & 47.9 & 28.1 & 7.7 \\
\hline Domestic debt & 27.5 & 36.8 & 24.4 & 16.8 & 5.4 \\
\hline External debt ${ }^{2 /}$ & 33.5 & 37.6 & 23.5 & 11.3 & 2.3 \\
\hline Debt-service due (commitments) & 13.2 & 17.5 & 7.6 & 3.6 & 1.1 \\
\hline Interest & 9.4 & 16.0 & 7.3 & 3.4 & 1.1 \\
\hline Repayments of principal ${ }^{3 /}$ & 3.8 & 1.5 & 0.3 & 0.2 & 0.0 \\
\hline Debt-service arrears & 0.0 & 0.4 & 4.5 & 3.3 & 0.9 \\
\hline Interest & 0.0 & 0.1 & 1.1 & 0.8 & 0.2 \\
\hline Repayments of principal ${ }^{3 /}$ & 0.0 & 0.2 & 3.4 & 2.5 & 0.7 \\
\hline \multicolumn{6}{|l|}{ Memorandum item: } \\
\hline GDP at market prices (in billions of Zimbabwe dollars) & 228 & 329 & 565 & 1,205 & 5,810 \\
\hline
\end{tabular}

Sources: Zimbabwean authorities; and IMF staff estimates.

1/ At cost value.

2/ External debt account may differ according to treatment of certain publicly guaranteed debts.

3/ Medium- and long-term debt. 
Table 22. Zimbabwe: Money Supply, 1998-2003

(In billions of Zimbabwe dollars)

\begin{tabular}{|c|c|c|c|c|c|c|}
\hline & 1998 & 1999 & 2000 & 2001 & 2002 & 2003 \\
\hline Notes and coins in circulation & 4.3 & 6.9 & 9.5 & 24.7 & 77.9 & 433.2 \\
\hline Demand deposits with the banking system & 20.4 & 27.4 & 43.1 & 103.8 & 270.6 & $1,626.1$ \\
\hline Narrow money (M1) & 24.7 & 34.3 & 52.6 & 128.5 & 348.5 & $2,059.3$ \\
\hline Savings deposits with the banking system & 12.9 & 17.1 & 23.9 & 45.7 & 104.0 & 356.6 \\
\hline Fixed deposits of 30 days or less with the banking system & 5.6 & 7.0 & 23.2 & 22.7 & 96.6 & 569.4 \\
\hline Broad money (M2) & 43.1 & 58.4 & 99.7 & 196.9 & 549.0 & $2,985.3$ \\
\hline Fixed deposits of at least 30 days with the banking system & 13.5 & 15.1 & 17.9 & 41.4 & 81.9 & 255.0 \\
\hline Broad money (M3) & 56.6 & 73.5 & 117.6 & 238.3 & 631.0 & $3,240.3$ \\
\hline
\end{tabular}

Sources: Reserve Bank of Zimbabwe; and IMF staff estimates. 
Table 23. Zimbabwe: Monetary Survey, 1998-2003 ${ }^{1 /}$

(ln billions of Zimbabwe dollars, unless otherwise indicated)

\begin{tabular}{|c|c|c|c|c|c|c|}
\hline & 1998 & 1999 & 2000 & 2001 & 2002 & 2003 \\
\hline Net foreign assets ${ }^{2 /}$ & -27.4 & -15.3 & -12.0 & -12.3 & -12.4 & -142.8 \\
\hline $\mathrm{RBZ}$ & -18.9 & -11.9 & -11.5 & -13.0 & -14.7 & -242.5 \\
\hline Deposit money banks and $\mathrm{OBIs} \mathrm{s}^{3 /}$ & -8.5 & -3.4 & -0.5 & 0.7 & 2.3 & 99.7 \\
\hline Net domestic assets & 84.0 & 88.8 & 129.6 & 250.6 & 643.3 & $3,383.1$ \\
\hline Domestic credit & 76.3 & 89.4 & 144.4 & 260.4 & 648.9 & $3,229.0$ \\
\hline Claims on government & 16.2 & 22.2 & 48.3 & 95.3 & 193.9 & 474.1 \\
\hline RBZ & 7.2 & 3.9 & 4.6 & 27.6 & 97.2 & 346.1 \\
\hline Deposit money banks and OBIs ${ }^{3 /}$ & 9.0 & 18.2 & 43.6 & 67.8 & 96.7 & 128.0 \\
\hline Claims on public enterprises & 3.4 & 4.6 & 9.2 & 19.7 & 46.5 & 214.0 \\
\hline $\mathrm{RBZ}$ & 0.7 & 0.7 & 0.7 & 2.0 & 0.6 & 0.1 \\
\hline Deposit money banks and OBIs ${ }^{3 /}$ & 2.8 & 4.0 & 8.5 & 17.7 & 45.9 & 213.9 \\
\hline Claims on nonbank private sector & 56.7 & 62.6 & 87.0 & 145.3 & 408.4 & $2,540.9$ \\
\hline RBZ & 0.8 & 1.1 & 2.0 & 2.3 & 2.7 & 7.2 \\
\hline Deposit money banks and OBIs ${ }^{3 /}$ & 55.9 & 61.5 & 84.9 & 143.1 & 405.8 & $2,533.7$ \\
\hline Other items (net) & 7.7 & -0.6 & -14.8 & -9.8 & -5.6 & 154.0 \\
\hline Broad money (M3) & 56.6 & 73.5 & 117.6 & 238.3 & 631.0 & $3,240.3$ \\
\hline \multicolumn{7}{|l|}{ Memorandum items: } \\
\hline Reserve money & 11.0 & 17.8 & 20.6 & 54.7 & 148.2 & 733.5 \\
\hline Money multiplier ${ }^{4 /}$ & 5.1 & 3.0 & 3.1 & 3.1 & 3.3 & 4.4 \\
\hline Currency/deposit ratio ${ }^{5 /}$ & 8.1 & 10.3 & 8.7 & 11.5 & 14.1 & 15.4 \\
\hline Reserves/deposit ratio ${ }^{6 /}$ & 22.7 & 16.1 & 10.3 & 14.0 & 12.6 & 10.5 \\
\hline
\end{tabular}

Sources: Reserve Bank of Zimbabwe; and IMF staff estimates.

1/ The coverage of all monetary aggregates (M1, M2, and M3) includes "Other banking institutions" (OBIs).

2/ Reserve Bank of Zimbabwe's net foreign assets and net domestic assets have been adjusted for memorandum of deposits.

3/ Includes commercial banks, discount houses, finance houses, and building societies.

4/ Defined as money supply (M3) divided by reserve money.

5/ Defined as notes and coins in circulation divided by total deposits.

6/ Defined as reserves held by deposit money banks at the RBZ divided by their total deposit liabilities. 
Table 24. Zimbabwe: Assets and Liabilities of Monetary Authorities, 1998-2003 ${ }^{1 /}$ (ln billions of Zimbabwe dollars)

\begin{tabular}{|c|c|c|c|c|c|c|}
\hline & 1998 & 1999 & 2000 & 2001 & 2002 & 2003 \\
\hline Net foreign assets ${ }^{1 / 2 /}$ & -18.9 & -11.9 & -11.5 & -13.0 & -14.7 & -242.5 \\
\hline Foreign assets & 11.0 & 18.2 & 15.6 & 6.7 & 7.1 & 108.7 \\
\hline Gold & 6.1 & 8.0 & 5.0 & 3.0 & 2.5 & 38.4 \\
\hline Foreign exchange & 4.8 & 10.2 & 10.6 & 3.6 & 4.6 & 70.0 \\
\hline SDR holdings & 0.0 & 0.0 & 0.0 & 0.1 & 0.0 & 0.2 \\
\hline Foreign liabilities & -29.9 & -30.1 & -27.1 & -19.8 & -21.8 & -351.2 \\
\hline Of which: Fund credit & -15.1 & -14.1 & -15.4 & -14.4 & -15.3 & -250.1 \\
\hline Net claims on government & 7.2 & 3.9 & 4.6 & 27.6 & 97.2 & 346.1 \\
\hline Claims on government & 36.8 & 51.2 & 88.2 & 27.6 & 106.8 & 346.1 \\
\hline Loans and advances ${ }^{3 /}$ & 35.7 & 51.2 & 84.0 & 2.3 & 44.9 & 56.4 \\
\hline Treasury bills & 1.1 & 0.0 & 4.2 & 25.3 & 61.9 & 289.7 \\
\hline Government deposits ${ }^{3 /}$ & -29.6 & -47.3 & -83.6 & 0.0 & -9.6 & 0.0 \\
\hline Claims on nonfinancial public enterprises & 0.7 & 0.7 & 0.7 & 2.0 & 0.6 & 0.1 \\
\hline Net claims on private sector & 7.5 & 11.6 & 16.3 & 31.0 & 67.4 & 433.4 \\
\hline Claims on deposit money banks & 6.7 & 10.5 & 14.3 & 28.7 & 64.7 & 426.2 \\
\hline Claims on nonbank private sector & 0.8 & 1.1 & 2.0 & 2.3 & 2.7 & 7.2 \\
\hline Other items (net; asset + ) & 14.5 & 13.5 & 10.6 & 7.1 & -2.3 & 196.5 \\
\hline Reserve money & 11.0 & 17.8 & 20.6 & 54.7 & 148.2 & 733.5 \\
\hline Reserves of deposit money banks & 6.1 & 10.1 & 10.4 & 28.6 & 67.0 & 283.3 \\
\hline Reserves of other banking institutions ${ }^{4 /}$ & 0.5 & 0.6 & 0.7 & 1.3 & 2.7 & 9.9 \\
\hline Currency in circulation & 4.3 & 6.9 & 9.5 & 24.7 & 77.9 & 433.2 \\
\hline Nonbank deposits & 0.2 & 0.2 & 0.1 & 0.2 & 0.7 & 7.2 \\
\hline
\end{tabular}

Sources: Reserve Bank of Zimbabwe; and IMF staff estimates.

1/ Net foreign assets are valued at current exchange rates and reflect the Fund's records for transactions with the Fund.

2/ Reserve Bank of Zimbabwe's net foreign assets and net domestic assets have been adjusted for memorandum of deposits. 3/ The RBZ reconfigured the loans and advances and government deposits series; this has been reflected in the 2000-01 figures.

4/ Includes finance houses, and building societies. 
Table 25. Zimbabwe: Consolidated Accounts of Deposit Money Banks and Other Banking Institutions, 1998-2003 ${ }^{1 /}$ (ln billions of Zimbabwe dollars)

\begin{tabular}{|c|c|c|c|c|c|c|}
\hline & 1998 & 1999 & 2000 & 2001 & 2002 & 2003 \\
\hline Net foreign assets & -8.5 & -3.4 & -0.5 & 0.7 & 2.3 & 99.7 \\
\hline Foreign assets & 6.7 & 6.0 & 9.0 & 8.0 & 12.7 & 180.4 \\
\hline Foreign liabilities & -15.1 & -9.3 & -9.3 & -7.2 & -10.3 & -80.7 \\
\hline Reserves & 6.5 & 10.7 & 10.4 & 31.9 & 91.5 & 620.3 \\
\hline Of which : currency & 0.6 & 1.3 & 1.7 & 4.2 & 16.2 & 96.9 \\
\hline Net credit from Reserve Bank & -6.7 & -10.5 & -14.7 & -19.1 & -61.2 & -473.9 \\
\hline Net claims on government & 9.0 & 18.2 & 43.6 & 67.8 & 96.7 & 128.0 \\
\hline Claims on government & 9.9 & 18.4 & 45.3 & 70.9 & 121.2 & 184.7 \\
\hline Treasury bills & 9.7 & 18.1 & 44.5 & 69.4 & 114.5 & 170.8 \\
\hline Government stock & 0.2 & 0.1 & 0.0 & 0.8 & 6.2 & 6.9 \\
\hline Loans and advances & 0.1 & 0.2 & 0.8 & 0.7 & 0.5 & 7.1 \\
\hline Government deposits & -0.9 & -0.2 & -1.7 & -3.1 & -24.5 & -56.7 \\
\hline Claims on nonfinancial public enterprises & 2.8 & 4.0 & 8.5 & 17.7 & 45.9 & 213.9 \\
\hline Claims on private sector & 55.9 & 61.5 & 84.9 & 143.1 & 405.8 & $2,533.7$ \\
\hline Loans and advances & 52.7 & 55.3 & 69.6 & 95.5 & 270.0 & $1,640.8$ \\
\hline Of which: on-lent external borrowing & 12.7 & 7.2 & 7.3 & 5.3 & 5.7 & 33.4 \\
\hline Bills discounted & 1.4 & 1.6 & 6.9 & 26.9 & 78.5 & 575.9 \\
\hline Bankers' acceptances & 0.6 & 2.0 & 3.6 & 6.6 & 37.1 & 198.5 \\
\hline Other investments & 1.2 & 2.6 & 4.8 & 14.0 & 20.2 & 118.4 \\
\hline Other items (net; asset + ) & -6.8 & -14.1 & -24.3 & -28.6 & -28.6 & -321.9 \\
\hline Deposits & 52.2 & 66.4 & 108.0 & 213.5 & 552.4 & $2,799.9$ \\
\hline
\end{tabular}

Sources: Reserve Bank of Zimbabwe; and IMF staff estimates.

1/ Includes commercial banks, merchant banks, discount houses, and acceptance houses. 
Table 26. Zimbabwe: Required Reserves and Liquid Asset Ratios, 1998-2003

(In percent of liabilities to the public)

\begin{tabular}{|c|c|c|c|c|c|c|}
\hline & 1998 & 1999 & 2000 & 2001 & 2002 & 2003 \\
\hline \multicolumn{7}{|l|}{ Required reserve ratios } \\
\hline \multicolumn{7}{|l|}{ Deposit money banks ${ }^{1 /}$} \\
\hline Demand deposits & 25.0 & 30.0 & 30.0 & 50.0 & 50.0 & 50.0 \\
\hline Savings \& time deposits & 25.0 & 30.0 & 30.0 & 20.0 & 20.0 & 20.0 \\
\hline Finance houses ${ }^{2 /}$ & 5.0 & 5.0 & 5.0 & 5.0 & 5.0 & 5.0 \\
\hline \multicolumn{7}{|l|}{ Liquid asset ratios ${ }^{3 /}$} \\
\hline \multicolumn{7}{|l|}{ Commercial banks } \\
\hline Prescribed & 10.0 & 10.0 & 10.0 & 10.0 & 10.0 & 10.0 \\
\hline Actual & 23.2 & 31.0 & 34.6 & 35.8 & 37.0 & 32.9 \\
\hline \multicolumn{7}{|l|}{ Accepting houses } \\
\hline Prescribed & 10.0 & 10.0 & 10.0 & 10.0 & 10.0 & 10.0 \\
\hline Actual & 86.7 & 116.0 & 76.7 & 44.0 & 38.0 & 27.0 \\
\hline \multicolumn{7}{|l|}{ Finance houses } \\
\hline Prescribed & 10.0 & 10.0 & 10.0 & 10.0 & 10.0 & 10.0 \\
\hline Actual & 13.0 & 18.0 & 29.2 & 35.0 & 29.0 & 90.0 \\
\hline \multicolumn{7}{|l|}{ Building societies } \\
\hline Prescribed & 10.0 & 10.0 & 10.0 & 10.0 & 10.0 & 10.0 \\
\hline Actual & 50.6 & 62.0 & 86.5 & 70.2 & 69.0 & 74.0 \\
\hline
\end{tabular}

Source: Reserve Bank of Zimbabwe.

1/ With effect from 1 February 2001, commercial and merchant banks are required to keep

50 percent of their demand deposits and 20 percent of their savings \& time deposits as required reserves.

2/ Base for requirements since May 1981 is liabilities to the public.

3 / The liquid asset ratio is defined as the ratio of liquid assets to the liabilities to the public. Liquid assets consist of the following: (i) notes and coins; (ii) balance with the Reserve Bank and discount houses; (iii) short-term debt (treasury, trade, and agricultural marketing authority); (iv) government and municipal stocks with less that one year remaining to maturity; and (v) net external positions. 
Table 27. Zimbabwe: Selected Interest Rates, 1998-2003 ${ }^{1 /}$

(In percent per year; end of period)

\begin{tabular}{|c|c|c|c|c|c|c|}
\hline & 1998 & 1999 & 2000 & 2001 & 2002 & 2003 \\
\hline Rediscount rate (maximum) ${ }^{2 /}$ & 39.50 & 74.41 & 63.30 & 57.20 & 57.20 & $\ldots$ \\
\hline Treasury bills (90 days) & 35.19 & 69.41 & 61.24 & 25.94 & 25.92 & 79.75 \\
\hline Call money (maximum) & 37.00 & 87.00 & 57.00 & 59.70 & 50.00 & 600.00 \\
\hline \multicolumn{7}{|l|}{ Certificates of deposit (maximum) } \\
\hline 3 month & 42.00 & 80.00 & 65.00 & 36.00 & 42.00 & 600.00 \\
\hline 6 month & 47.00 & 80.00 & 80.00 & 65.00 & 40.00 & 800.00 \\
\hline 12 month & 41.00 & 75.00 & 75.00 & 70.00 & 40.00 & 900.00 \\
\hline 24 month & 40.00 & 70.00 & 60.00 & 70.00 & 50.00 & 900.00 \\
\hline \multicolumn{7}{|l|}{ Savings accounts (maximum) } \\
\hline Commercial banks & 27.50 & 44.00 & 41.00 & 19.00 & 19.00 & 45.00 \\
\hline Building societies & 14.00 & 14.00 & 14.00 & 14.00 & 12.50 & 41.85 \\
\hline Post Office Savings Bank & 21.00 & 21.00 & 21.00 & 21.00 & 11.00 & 11.00 \\
\hline \multicolumn{7}{|l|}{ Fixed deposits } \\
\hline \multicolumn{7}{|l|}{ Commercial banks } \\
\hline 3 month & 40.00 & 50.00 & 46.25 & 15.00 & 19.50 & 350.00 \\
\hline 12 month & 39.00 & 48.50 & 41.75 & 20.50 & 25.00 & 375.00 \\
\hline 24 month & 32.00 & 39.75 & 34.75 & 20.00 & 26.50 & 385.00 \\
\hline \multicolumn{7}{|l|}{ Acceptance houses } \\
\hline 3 month & 40.00 & 70.50 & 61.00 & 24.75 & 37.00 & 550.00 \\
\hline 12 month & 35.25 & 51.25 & 40.00 & 27.50 & 31.75 & 450.00 \\
\hline 24 month & 34.75 & 34.88 & 30.00 & 26.50 & 26.00 & 444.00 \\
\hline \multicolumn{7}{|l|}{ Finance houses } \\
\hline 3 month & 40.25 & 64.00 & 47.50 & 18.50 & 28.00 & 600.00 \\
\hline 12 month & 42.00 & 57.00 & 52.00 & 25.50 & 35.00 & 300.00 \\
\hline 24 month & 35.00 & 54.50 & 51.50 & $\ldots$ & $\ldots$ & $\ldots$ \\
\hline \multicolumn{7}{|l|}{ Post Office Savings Bank } \\
\hline 12 month & 24.00 & 24.00 & 24.00 & 15.00 & 15.00 & 15.00 \\
\hline \multicolumn{7}{|l|}{ Average cost of funds } \\
\hline Commercial banks & 15.75 & 21.84 & 26.47 & 11.27 & 13.44 & 88.52 \\
\hline \multicolumn{7}{|l|}{ Lending rates } \\
\hline Commercial banks (minimum) & 40.50 & 56.00 & 55.00 & 15.00 & 35.00 & 82.00 \\
\hline Commercial banks (weighted average) & 49.25 & 66.00 & 68.25 & 31.25 & 45.75 & 346.00 \\
\hline Building societies (low-cost housing) & 24.50 & 21.50 & 28.75 & 25.88 & 30.75 & 44.50 \\
\hline
\end{tabular}

Source: Reserve Bank of Zimbabwe.

$1 /$ Rates are quoted as simple annual rates.

2/ On December 1, 1998, the rediscount rate was replaced by a Reserve Bank rate, which was suspended on November 20, 2002. 
Table 28. Zimbabwe: Sectoral Anaysis of Commercial Banks' Loans and Advances, 1998-2003

\begin{tabular}{|c|c|c|c|c|c|c|}
\hline & 1998 & 1999 & 2000 & 2001 & 2002 & 2003 \\
\hline & \multicolumn{6}{|c|}{ (In millions of Zimbabwe dollars) } \\
\hline Total & 25,008 & 36,567 & 55,089 & 98,545 & 258,054 & $1,467,120$ \\
\hline Agriculture & 4,052 & 5,772 & 7,962 & 12,643 & 28,916 & 192,683 \\
\hline Construction & 575 & 553 & 2,347 & 1,863 & 5,873 & 10,704 \\
\hline Communications & 333 & 588 & 409 & 2,983 & 3,467 & 23,631 \\
\hline Distribution & 5,668 & 6,358 & 9,089 & 27,371 & 61,383 & 395,042 \\
\hline Financial and investments & 1,120 & 1,121 & 6,677 & 10,602 & 12,920 & 96,191 \\
\hline Financial organizations & 439 & 972 & 4,096 & 1,552 & 17,447 & 109,667 \\
\hline Manufacturing & 4,182 & 6,447 & 7,712 & 20,295 & 53,864 & 299,197 \\
\hline Mining & 633 & 2,641 & 5,370 & 4,332 & 6,403 & 18,754 \\
\hline Services & 5,041 & 7,264 & 6,232 & 6,597 & 36,105 & 144,268 \\
\hline Transport & 787 & 1,219 & 1,847 & 2,275 & 8,509 & 62,075 \\
\hline Individuals & 2,169 & 3,193 & 3,103 & 6,408 & 20,088 & 110,449 \\
\hline \multirow[t]{2}{*}{ Other } & 9 & 439 & 245 & 1,624 & 3,079 & 4,459 \\
\hline & \multicolumn{6}{|c|}{ (In percent of total) } \\
\hline Total & 100.0 & 100.0 & 100.0 & 100.0 & 100.0 & 100.0 \\
\hline Agriculture & 16.2 & 15.8 & 14.5 & 12.8 & 11.2 & 13.1 \\
\hline Construction & 2.3 & 1.5 & 4.3 & 1.9 & 2.3 & 0.7 \\
\hline Communications & 1.3 & 1.6 & 0.7 & 3.0 & 1.3 & 1.6 \\
\hline Distribution & 22.7 & 17.4 & 16.5 & 27.8 & 23.8 & 26.9 \\
\hline Financial and investments & 4.5 & 3.1 & 12.1 & 10.8 & 5.0 & 6.6 \\
\hline Financial organizations & 1.8 & 2.7 & 7.4 & 1.6 & 6.8 & 7.5 \\
\hline Manufacturing & 16.7 & 17.6 & 14.0 & 20.6 & 20.9 & 20.4 \\
\hline Mining & 2.5 & 7.2 & 9.7 & 4.4 & 2.5 & 1.3 \\
\hline Services & 20.2 & 19.9 & 11.3 & 6.7 & 14.0 & 9.8 \\
\hline Transport & 3.1 & 3.3 & 3.4 & 2.3 & 3.3 & 4.2 \\
\hline Individuals & 8.7 & 8.7 & 5.6 & 6.5 & 7.8 & 7.5 \\
\hline Other & 0.0 & 1.2 & 0.4 & 1.6 & 1.2 & 0.3 \\
\hline
\end{tabular}

Source: Reserve Bank of Zimbabwe. 
Table 29. Zimbabwe: Sectoral Anaysis of Merchant Banks' Loans and Advances, 1998-2002

\begin{tabular}{|c|c|c|c|c|c|c|}
\hline & 1998 & 1999 & 2000 & 2001 & 2001 & 2002 \\
\hline & \multicolumn{6}{|c|}{ (In millions of Zimbabwe dollars) } \\
\hline Total & 11,671 & 7,037 & 8,449 & 12,468 & 56,061 & 396,879 \\
\hline Agriculture & 926 & 826 & 523 & 604 & 4,390 & 14,463 \\
\hline Distribution & 4,392 & 1,547 & 2,049 & 2,381 & 9,676 & 187,698 \\
\hline Financial and investments & 164 & 58 & 15 & 0 & 712 & 6,530 \\
\hline Financial organizations & 200 & 143 & 174 & 430 & 445 & 1,509 \\
\hline Manufacturing & 2,358 & 2,017 & 2,471 & 4,805 & 22,033 & 104,197 \\
\hline Mining & 1,732 & 681 & 1,207 & 772 & 2,983 & 5,501 \\
\hline Services & 822 & 570 & 757 & 1,170 & 6,272 & 45,649 \\
\hline Transport & 186 & 235 & 217 & 415 & 1,709 & 2,563 \\
\hline Individuals & 135 & 167 & 128 & 155 & 824 & 17,055 \\
\hline \multirow[t]{2}{*}{ Other } & 756 & 793 & 908 & 1,736 & 7,017 & 11,714 \\
\hline & \multicolumn{6}{|c|}{ (In percent of total) } \\
\hline Total & 100.0 & 100.0 & 100.0 & 100.0 & 100.0 & 100.0 \\
\hline Agriculture & 7.9 & 11.7 & 6.2 & 4.8 & 7.8 & 3.6 \\
\hline Distribution & 37.6 & 22.0 & 24.3 & 19.1 & 17.3 & 47.3 \\
\hline Financial and investments & 1.4 & 0.8 & 0.2 & 0.0 & 1.3 & 1.6 \\
\hline Financial organizations & 1.7 & 2.0 & 2.1 & 3.4 & 0.8 & 0.4 \\
\hline Manufacturing & 20.2 & 28.7 & 29.2 & 38.5 & 39.3 & 26.3 \\
\hline Mining & 14.8 & 9.7 & 14.3 & 6.2 & 5.3 & 1.4 \\
\hline Services & 7.0 & 8.1 & 9.0 & 9.4 & 11.2 & 11.5 \\
\hline Transport & 1.6 & 3.3 & 2.6 & 3.3 & 3.0 & 0.6 \\
\hline Individuals & 1.2 & 2.4 & 1.5 & 1.2 & 1.5 & 4.3 \\
\hline Other & 6.5 & 11.3 & 10.7 & 13.9 & 12.5 & 3.0 \\
\hline
\end{tabular}

Source: Reserve Bank of Zimbabwe. 
Table 30. Zimbabwe: Nonbank Financial Institutions' Assets, 1998-2003 (ln millions of Zimbabwe dollars, unless otherwise indicated)

\begin{tabular}{|c|c|c|c|c|c|c|}
\hline & 1998 & 1999 & 2000 & 2001 & 2002 & 2003 \\
\hline Post Office Savings Bank, total assets & 6,662 & 7,325 & 8,016 & 14,292 & 22,189 & 57,092 \\
\hline Claims on government & 1,542 & 1,839 & 2,075 & 2,791 & 3,843 & 4,486 \\
\hline Claims on public enterprises & 859 & 1,388 & 1,138 & 1,764 & 2,148 & 1,522 \\
\hline Claims on the private sector & 889 & 942 & 942 & 1,035 & 1,049 & 1,064 \\
\hline lnterbank NCDs and call money ${ }^{1 /}$ & 2,107 & 1,888 & 2,385 & 7,222 & 13,640 & 46,259 \\
\hline Other assets & 0 & 0 & 0 & 0 & 176 & 1,347 \\
\hline Reserves & 1,265 & 1,270 & 1,478 & 1,479 & 1,333 & 2,414 \\
\hline Building societies, total assets & 18,054 & 24,324 & 32,402 & 52,572 & 107,556 & 333,204 \\
\hline Claims on government & 1,955 & 6,928 & 11,542 & 14,556 & 7,260 & 7,663 \\
\hline Claims on public enterprises & 220 & 44 & 30 & 106 & 100 & 100 \\
\hline Claims on the private sector & 11,128 & 10,917 & 11,271 & 16,843 & 35,575 & 104,142 \\
\hline lnterbank NCDs and call money ${ }^{1 /}$ & 2,312 & 2,890 & 5,003 & 10,714 & 32,617 & 63,807 \\
\hline Other assets & 1,487 & 2,940 & 3,152 & 5,458 & 12,723 & 64,597 \\
\hline Reserves & 953 & 606 & 1,404 & 4,896 & 19,282 & 92,895 \\
\hline Finance houses, total assets & 6,983 & 6,899 & 7,056 & 14,657 & 40,960 & 104,192 \\
\hline Claims on government & 554 & 467 & 456 & 808 & 1,646 & 5,293 \\
\hline Claims on public enterprises & 26 & 40 & 0 & 143 & 0 & 0 \\
\hline Claims on the private sector & 5,888 & 5,806 & 5,595 & 9,269 & 33,090 & 67,387 \\
\hline lnterbank NCDs and call money ${ }^{1 /}$ & 57 & 121 & 158 & 196 & 1,593 & 5,280 \\
\hline Other assets & 172 & 197 & 519 & 815 & 2,200 & 14,386 \\
\hline Reserves & 262 & 213 & 268 & 3,385 & 2,431 & 11,846 \\
\hline Foreign assets & 24 & 55 & 61 & 41 & 0 & 0 \\
\hline \multicolumn{7}{|l|}{ Consolidated other banking institutions, } \\
\hline Total assets & 31,697 & 38,548 & 47,475 & 65,448 & 132,562 & 307,003 \\
\hline Claims on government & 4,050 & 9,233 & 14,072 & 18,156 & 12,749 & 17,442 \\
\hline Claims on public enterprises & 1,105 & 1,472 & 1,168 & 2,013 & 2,248 & 1,622 \\
\hline Claims on the private sector & 17,905 & 17,665 & 17,808 & 27,147 & 69,714 & 172,593 \\
\hline lnterbank NCDs and call money ${ }^{1 /}$ & 4,475 & 4,899 & 7,546 & 18,132 & 47,850 & 115,346 \\
\hline Other assets & 4,162 & 5,279 & 6,881 & 0 & 0 & 0 \\
\hline \multicolumn{7}{|l|}{ Insurance and pension funds, total } \\
\hline Asset base & 42,706 & 51,683 & 63,044 & 77,727 & 123,683 & 220,709 \\
\hline Life insurance & 27,441 & 34,679 & 42,337 & 50,825 & 76,745 & 151,120 \\
\hline Professional life reinsurance & 246 & 396 & 316 & 356 & 619 & 5,772 \\
\hline Nonlife insurance & 1,794 & 1,280 & 1,646 & 2,307 & 4,335 & 7,881 \\
\hline Professional nonlife reinsurance & 2,144 & 1,723 & 1,573 & 2,577 & 4,929 & 9,242 \\
\hline Self-administered pension funds & 11,081 & 13,605 & 17,172 & 21,662 & 37,055 & 46,694 \\
\hline Prescribed securities & 9,597 & 10,635 & 12,479 & 15,211 & 22,856 & 28,063 \\
\hline Life insurance & 5,210 & 6,659 & 7,483 & 7,864 & 7,539 & 12,701 \\
\hline Professional life reinsurance & 118 & 222 & 241 & 288 & 420 & 448 \\
\hline Nonlife insurance & 247 & 445 & 714 & 756 & 1,460 & 2,231 \\
\hline Professional nonlife reinsurance & 102 & 435 & 664 & 699 & 1,133 & 1,628 \\
\hline Self-administered pension funds & 3,920 & 2,874 & 3,377 & 5,604 & 12,304 & 11,056 \\
\hline \multicolumn{7}{|l|}{ Total nonbank financial sector } \\
\hline Total assets & 42,706 & 51,683 & 63,044 & 77,727 & 123,683 & 220,709 \\
\hline Of which: claims on government & 9,597 & 10,635 & 12,479 & 15,211 & 22,856 & 28,063 \\
\hline Share in total (in percent) & 22.5 & 20.6 & 19.8 & 19.6 & 18.5 & 12.7 \\
\hline
\end{tabular}

Source: Reserve Bank of Zimbabwe.

1/ NCDs are negotiable certificates of deposit. 
Table 31. Zimbabwe: Balance of Payments, 1998-2003

(In millions of U.S. dollars, unless otherwise indicated)

\begin{tabular}{|c|c|c|c|c|c|c|}
\hline & 1998 & 1999 & 2000 & 2001 & 2002 & $\begin{array}{r}2003 \\
\text { Est. }\end{array}$ \\
\hline Current account balance (excluding official transfers) & -372 & 47 & -38 & -497 & -604 & -421 \\
\hline Trade balance & -95 & 249 & 293 & -217 & -525 & -402 \\
\hline Exports, f.o.b. & 1,925 & 1,924 & 2,200 & 1,575 & 1,398 & 1,225 \\
\hline Imports, f.o.b. & $-2,020$ & $-1,675$ & $-1,907$ & $-1,792$ & $-1,923$ & $-1,627$ \\
\hline Of which : emergency food imports & 0 & 0 & 0 & -68 & -337 & -281 \\
\hline Nonfactor services & -59 & -2 & -164 & -198 & -203 & -250 \\
\hline Receipts & 630 & 621 & 331 & 243 & 210 & 184 \\
\hline Payments & -689 & -623 & -495 & -441 & -412 & -433 \\
\hline Investment income & -385 & -355 & -358 & -331 & -274 & -278 \\
\hline Interest & -184 & -140 & -142 & -147 & -155 & -171 \\
\hline Receipts & 30 & 37 & 26 & 12 & 10 & 8 \\
\hline Payments & -214 & -176 & -168 & -159 & -165 & -179 \\
\hline Other (net) & -201 & -215 & -217 & -184 & -119 & -107 \\
\hline Private transfers (net) & 166 & 155 & 191 & 249 & 398 & 508 \\
\hline Capital account (including official transfers) & 560 & 143 & -227 & -396 & -304 & -256 \\
\hline Official transfers (net) & 77 & 101 & 53 & 40 & 35 & 35 \\
\hline Direct investment (net) & 436 & 50 & 16 & 0 & 23 & 5 \\
\hline Portfolio investment (net) & 11 & 21 & -1 & -68 & -2 & 11 \\
\hline Long-term capital (net) & 96 & -3 & -155 & -282 & -279 & -227 \\
\hline Short-term capital (net) & -60 & -26 & -140 & -86 & -81 & -79 \\
\hline Errors and omissions & -229 & -227 & 130 & 620 & 269 & 103 \\
\hline Overall balance & -41 & -37 & -136 & -273 & -639 & -574 \\
\hline Financing & 41 & 37 & 136 & 273 & 639 & 574 \\
\hline Gross official reserves (- increase) & 64 & 8 & 25 & 2 & 5 & -3 \\
\hline Arrears (- decrease) & 0 & 114 & 281 & 399 & 717 & 621 \\
\hline Net use of Fund resources & 5 & -28 & -70 & -85 & -95 & -65 \\
\hline Net other liabilities & -27 & -57 & -106 & -44 & 13 & 21 \\
\hline \multicolumn{7}{|l|}{ Memorandum items: } \\
\hline Gross official international reserves ${ }^{1 /}$ & 55 & 47 & 22 & 20 & 15 & 18 \\
\hline In months of imports of goods and services & 0.3 & 0.2 & 0.1 & 0.1 & 0.1 & 0.1 \\
\hline Current account balance (in percent of GDP) $)^{2 /}$ & -4.0 & 0.5 & -0.4 & -5.9 & -7.9 & -6.0 \\
\hline
\end{tabular}

Sources: Zimbabwean authorities; and IMF staff estimates.

1/ End of period; usable reserves.

2/ GDP at world prices using real GDP growth and trading partner countries' inflation (base year is 1996). 
Table 32. Zimbabwe: External Trade Indicators, 1998-2003

$(1990=100$, unless otherwise indicated)

\begin{tabular}{|c|c|c|c|c|c|c|}
\hline & 1998 & 1999 & 2000 & 2001 & 2002 & $\begin{array}{r}2003 \\
\text { Est. }\end{array}$ \\
\hline \multicolumn{7}{|l|}{ Exports } \\
\hline Value (in U.S. dollars terms) & 109.8 & 109.8 & 125.5 & 89.9 & 79.7 & 69.9 \\
\hline Percent change & -20.6 & 0.0 & 14.3 & -28.4 & -11.3 & -12.3 \\
\hline Volume & 109.4 & 114.9 & 122.2 & 96.5 & 83.0 & 68.7 \\
\hline Percent change & -10.4 & 5.0 & 6.3 & -21.1 & -13.9 & -17.3 \\
\hline Unit value (in U.S. dollars terms) & 100.3 & 95.5 & 102.7 & 93.1 & 96.0 & 101.8 \\
\hline Percent change & -11.3 & -4.8 & 7.5 & -9.3 & 3.1 & 6.0 \\
\hline \multicolumn{7}{|l|}{ Imports } \\
\hline Value (in U.S. dollars terms) & 133.7 & 110.9 & 126.2 & 118.6 & 127.3 & 107.7 \\
\hline Percent change & -23.9 & -17.1 & 13.9 & -6.0 & 7.3 & -15.4 \\
\hline Volume & 156.5 & 127.6 & 138.4 & 137.1 & 143.4 & 112.6 \\
\hline Percent change & -14.7 & -18.5 & 8.5 & -0.9 & 4.6 & -21.5 \\
\hline Unit value (in U.S. dollars terms) & 85.4 & 86.9 & 91.2 & 86.5 & 88.7 & 95.7 \\
\hline Percentage change & -10.8 & 1.7 & 5.0 & -5.2 & 2.6 & 7.8 \\
\hline Terms of trade & 117.5 & 109.9 & 112.6 & 107.7 & 108.2 & 106.4 \\
\hline Percent change & -0.6 & -6.4 & 2.5 & -4.4 & 0.5 & -1.7 \\
\hline
\end{tabular}

Sources: Reserve Bank of Zimbabwe; and IMF staff estimates. 
Table 33. Zimbabwe: Exports by Commodity, $1998-2003^{1 /}$ (continued)

(Values in millions of U.S. dollars; volumes in thousands of kilograms, unless otherwise indicated)

\begin{tabular}{|c|c|c|c|c|c|c|}
\hline & 1998 & 1999 & 2000 & 2001 & 2002 & $\begin{array}{r}2003 \\
\text { Est. }\end{array}$ \\
\hline Agricultural exports & 805.5 & 844.4 & 855.8 & 832.8 & 646.6 & 516.6 \\
\hline Tobacco & 523.8 & 612.0 & 548.7 & 594.3 & 434.7 & 321.0 \\
\hline Volume & 173.3 & 216.2 & 180.4 & 198.2 & 143.5 & 103.2 \\
\hline Unit value & 3.0 & 2.8 & 3.0 & 3.0 & 3.0 & 3.1 \\
\hline Sugar & 62.9 & 51.5 & 96.4 & 70.0 & 64.2 & 54.9 \\
\hline Volume & 200.9 & 162.6 & 248.2 & 179.5 & 150.0 & 113.5 \\
\hline Unit value & 0.3 & 0.3 & 0.4 & 0.4 & 0.4 & 0.5 \\
\hline Maize & 46.4 & 9.1 & 2.5 & 0.0 & 0.0 & 0.0 \\
\hline Volume & 311.7 & 75.8 & 20.8 & 0.0 & 0.0 & 0.0 \\
\hline Unit value & 0.1 & 0.1 & 0.1 & 0.1 & 0.1 & 0.1 \\
\hline Cold Storage Company beef & 32.1 & 32.6 & 39.7 & 22.7 & 2.3 & 0.6 \\
\hline Volume & 8.5 & 8.7 & 11.3 & 7.7 & 0.5 & 0.4 \\
\hline Unit value & 3.8 & 3.8 & 3.5 & 2.9 & 4.6 & 1.7 \\
\hline Coffee & 51.2 & 37.2 & 17.1 & 15.0 & 5.4 & 5.0 \\
\hline Volume & 12.7 & 10.0 & 6.5 & 6.2 & 4.9 & 4.5 \\
\hline Unit value & 4.0 & 3.7 & 2.6 & 2.4 & 1.1 & 1.1 \\
\hline Horticulture & 67.0 & 82.6 & 125.4 & 118.9 & 126.6 & 117.9 \\
\hline Volume & 40.1 & 48.6 & 33.8 & 39.9 & 40.3 & 37.9 \\
\hline Unit value & 1.7 & 1.7 & 3.7 & 3.0 & 3.1 & 3.1 \\
\hline Other agricultural & 22.2 & 19.4 & 26.1 & 12.0 & 13.4 & 17.3 \\
\hline Mineral exports & 395.2 & 387.0 & 440.4 & 390.8 & 297.8 & 370.6 \\
\hline Gold $^{2 /}$ & 236.1 & 229.7 & 216.4 & 225.8 & 159.5 & 137.4 \\
\hline Volume & 821.5 & 825.4 & 778.4 & 827.2 & 512.9 & 376.5 \\
\hline Unit value & 287.4 & 278.3 & 278.0 & 273.0 & 311.0 & 365.0 \\
\hline Asbestos & 36.1 & 35.6 & 61.1 & 60.0 & 39.3 & 42.4 \\
\hline Volume & 112.9 & 113.0 & 134.1 & 129.0 & 151.9 & 170.2 \\
\hline Unit value & 0.3 & 0.3 & 0.5 & 0.5 & 0.3 & 0.2 \\
\hline Nickel & 44.2 & 48.1 & 77.9 & 35.2 & 31.8 & 78.3 \\
\hline Volume & 10.1 & 8.1 & 9.0 & 6.3 & 4.9 & 8.9 \\
\hline Unit value & 4.4 & 5.9 & 8.7 & 5.6 & 6.5 & 8.8 \\
\hline Platinum & 3.4 & 3.5 & 11.4 & 17.5 & 14.5 & 68.2 \\
\hline Volume & 9.4 & 9.4 & 21.1 & 35.6 & 11.6 & 40.7 \\
\hline Unit value & 0.4 & 0.4 & 0.5 & 0.5 & 1.2 & 1.7 \\
\hline
\end{tabular}


Table 33. Zimbabwe: Exports by Commodity, 1998-2003 ${ }^{1 /}$ (concluded) (Values in millions of U.S. dollars; volumes in thousands of kilograms, unless otherwise indicated)

\begin{tabular}{|c|c|c|c|c|c|c|}
\hline & 1998 & 1999 & 2000 & 2001 & 2002 & $\begin{array}{r}2003 \\
\text { Est. }\end{array}$ \\
\hline Copper & 4.1 & 5.3 & 8.0 & 0.6 & 8.9 & 3.4 \\
\hline Volume & 2.6 & 3.4 & 4.4 & 0.5 & 2.6 & 2.6 \\
\hline Unit value & 1.6 & 1.6 & 1.8 & 1.2 & 3.4 & 1.3 \\
\hline Other mineral & 58.1 & 64.8 & 65.5 & 51.7 & 43.8 & 40.9 \\
\hline Manufacturing exports & 629.4 & 609.1 & 814.9 & 313.5 & 287.3 & 265.9 \\
\hline Ferrous alloys & 143.1 & 152.1 & 154.8 & 81.8 & 106.8 & 105.2 \\
\hline Volume & 233.5 & 236.9 & 274.0 & 222.2 & 306.8 & 295.6 \\
\hline Unit value & 0.6 & 0.6 & 0.6 & 0.4 & 0.3 & 0.4 \\
\hline Cotton lint & 150.1 & 111.9 & 156.0 & 81.9 & 53.2 & 50.2 \\
\hline Volume & 79.7 & 84.2 & 114.0 & 80.0 & 52.2 & 48.0 \\
\hline Unit value & 1.9 & 1.3 & 1.4 & 1.0 & 1.0 & 1.0 \\
\hline Iron and steel & 8.3 & 12.5 & 15.0 & 3.5 & 22.3 & 16.5 \\
\hline Volume & 36.8 & 55.0 & 65.2 & 15.3 & 78.8 & 60.0 \\
\hline Unit value & 0.2 & 0.2 & 0.2 & 0.2 & 0.3 & 0.3 \\
\hline Textiles and clothing & 58.0 & 59.3 & 79.3 & 20.2 & 17.7 & 17.0 \\
\hline Machinery and equipment & 12.4 & 17.0 & 50.6 & 8.6 & 5.2 & 4.4 \\
\hline Chemicals & 25.8 & 27.3 & 64.3 & 5.8 & 3.5 & 2.5 \\
\hline Other manufacturing & 231.6 & 229.0 & 294.9 & 111.7 & 78.6 & 70.1 \\
\hline Total exports ${ }^{3 /}$ & $1,924.9$ & $1,924.5$ & $2,200.5$ & $1,575.2$ & $1,397.9$ & $1,225.4$ \\
\hline
\end{tabular}

Sources: Reserve Bank of Zimbabwe; Central Statistical Office; and IMF staff estimates.

$1 /$ At the official exchange rate.

2/ Volume in thousands of ounces and unit value in U.S. dollars per ounce.

3/ Excludes estimated unidentified exports and internal freight. 
Table 34. Zimbabwe: Direction of Export Trade, 1998-2002 (In percent of total exports)

\begin{tabular}{|c|c|c|c|c|c|}
\hline & 1998 & 1999 & 2000 & 2001 & 2002 \\
\hline Industrial countries & 48.7 & 50.3 & 46.7 & 56.5 & 52.7 \\
\hline Australia & 0.6 & 0.5 & 0.6 & 0.3 & 0.3 \\
\hline Austria & 0.5 & 0.3 & 0.3 & 0.8 & 0.2 \\
\hline Belgium & 3.1 & 1.5 & 1.0 & 1.1 & 0.8 \\
\hline Denmark & 0.5 & 0.7 & 0.8 & 1.3 & 0.3 \\
\hline France & 0.9 & 1.1 & 0.9 & 2.3 & 2.6 \\
\hline Germany & 7.7 & 8.5 & 7.8 & 12.9 & 7.5 \\
\hline Italy & 3.5 & 3.9 & 3.5 & 3.4 & 2.4 \\
\hline Japan & 4.8 & 7.7 & 7.3 & 9.7 & 9.5 \\
\hline Netherlands & 3.0 & 2.1 & 3.3 & 2.1 & 2.4 \\
\hline Portugal & 1.3 & 1.1 & 0.5 & 2.6 & 1.1 \\
\hline Spain & 2.3 & 2.6 & 1.7 & 2.7 & 5.1 \\
\hline Sweden & 0.6 & 0.7 & 0.6 & 0.5 & 0.1 \\
\hline Switzerland & 2.5 & 2.7 & 4.0 & 1.0 & 8.2 \\
\hline United Kingdom & 10.7 & 10.5 & 8.7 & 10.8 & 7.9 \\
\hline United States & 6.7 & 6.4 & 5.7 & 5.1 & 4.2 \\
\hline Developing countries & 31.5 & 33.2 & 32.7 & 22.2 & 32.4 \\
\hline Botswana & 4.4 & 3.9 & 3.2 & 1.1 & 2.0 \\
\hline China, People's Republic of & 1.0 & 5.9 & 5.2 & 8.7 & 1.5 \\
\hline Malawi & 3.6 & 4.2 & 2.5 & 0.9 & 3.7 \\
\hline Mozambique & 1.8 & 2.1 & 1.9 & 0.3 & 1.9 \\
\hline South Africa & 13.1 & 12.6 & 15.0 & 10.1 & 16.4 \\
\hline Democratic Republic of Congo & 0.5 & 0.5 & 0.2 & 0.4 & 0.8 \\
\hline Zambia & 7.2 & 4.0 & 4.3 & 1.0 & 6.2 \\
\hline Other industrial and developing countries & 19.8 & 16.5 & 20.6 & 21.2 & 14.9 \\
\hline Total & 100.0 & 100.0 & 100.0 & 100.0 & 100.0 \\
\hline
\end{tabular}

Sources: Central Statistical Office; and IMF, Direction of Trade Statistics. 
Table 35. Zimbabwe: Imports by Principal Commodities, 1998-2003 ${ }^{1 /}$

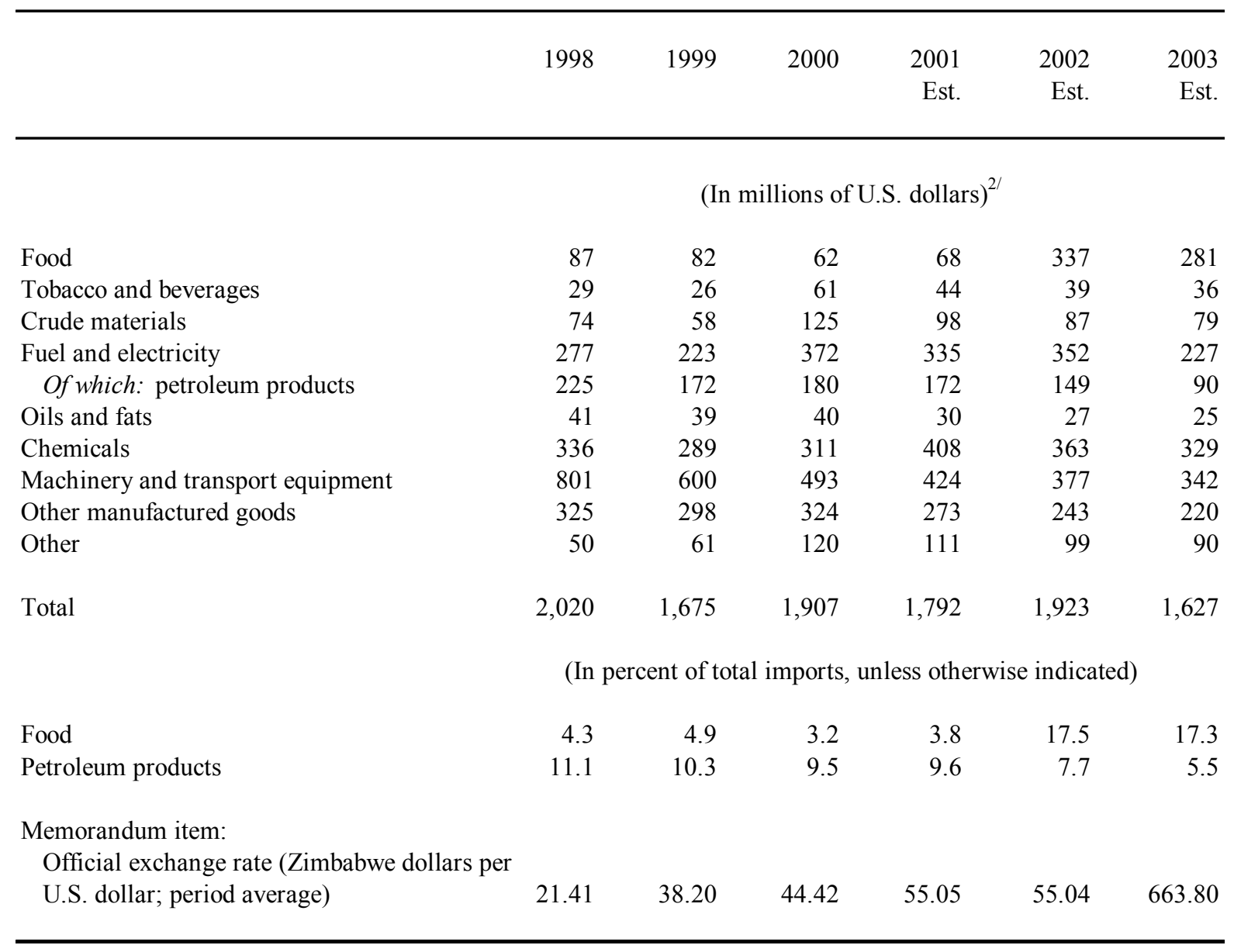

Sources: Central Statistics Office; and IMF staff estimates.

1/ On f.o.b. basis.

$2 /$ At the official exchange rate. 
Table 36. Zimbabwe: Direction of Import Trade, 1998-2002 (In percent of total imports)

\begin{tabular}{|c|c|c|c|c|c|}
\hline & 1998 & 1999 & 2000 & 2001 & 2002 \\
\hline Industrial countries & 31.6 & 29.4 & 25.5 & 15.4 & 14.4 \\
\hline Australia & 1.1 & 0.7 & 0.6 & 0.4 & 0.2 \\
\hline Austria & 0.5 & 0.3 & 0.3 & 0.6 & 0.1 \\
\hline Belgium & 1.0 & 1.4 & 1.0 & 0.6 & 0.4 \\
\hline Denmark & 0.4 & 0.4 & 0.3 & 0.2 & 0.2 \\
\hline France & 3.1 & 2.9 & 2.3 & 1.6 & 0.8 \\
\hline Germany & 3.7 & 5.2 & 3.1 & 2.8 & 3.2 \\
\hline Italy & 2.0 & 1.1 & 1.3 & 0.5 & 0.6 \\
\hline Japan & 4.8 & 4.0 & 4.1 & 1.7 & 2.3 \\
\hline Netherlands & 1.3 & 1.4 & 1.1 & 0.7 & 0.5 \\
\hline Norway & 0.3 & 0.2 & 0.2 & 0.1 & $\ldots$ \\
\hline Sweden & 0.5 & 0.5 & 0.4 & 0.3 & 0.4 \\
\hline United Kingdom & 6.8 & 6.5 & 4.8 & 3.2 & 2.9 \\
\hline United States & 5.8 & 4.7 & 6.0 & 2.8 & 2.9 \\
\hline Developing countries & 48.7 & 54.0 & 56.9 & 60.4 & 53.2 \\
\hline Botswana & 1.7 & 1.9 & 4.3 & 2.0 & 2.3 \\
\hline India & 1.4 & 1.5 & 1.1 & $\ldots$ & $\ldots$ \\
\hline Mozambique & $\ldots$ & $\ldots$ & $\ldots$ & 5.2 & 7.7 \\
\hline South Africa & 38.5 & 40.7 & 38.4 & 46.8 & 42.5 \\
\hline Taiwan Province of China & 1.1 & 0.9 & 0.9 & $\ldots$ & ... \\
\hline Democratic Republic of Congo & $\ldots$ & $\ldots$ & $\ldots$ & 5.6 & 0.2 \\
\hline Zambia & 0.8 & 0.9 & 1.0 & 0.8 & 0.5 \\
\hline Other (petroleum) $)^{1 /}$ & 5.3 & 8.1 & 11.2 & $\ldots$ & $\ldots$ \\
\hline Other industrial and developing countries & 19.7 & 16.6 & 17.6 & 24.2 & 32.4 \\
\hline Total & 100.0 & 100.0 & 100.0 & 100.0 & 100.0 \\
\hline
\end{tabular}

Sources: Central Statistical Office; and IMF, Direction of Trade Statistics .

1/ Petroleum imports of unknown origin transported through South Africa. 
Table 37. Zimbabwe: International Reserves, 1998-2003

(In millions of U.S. dollars, unless otherwise indicated; end of period)

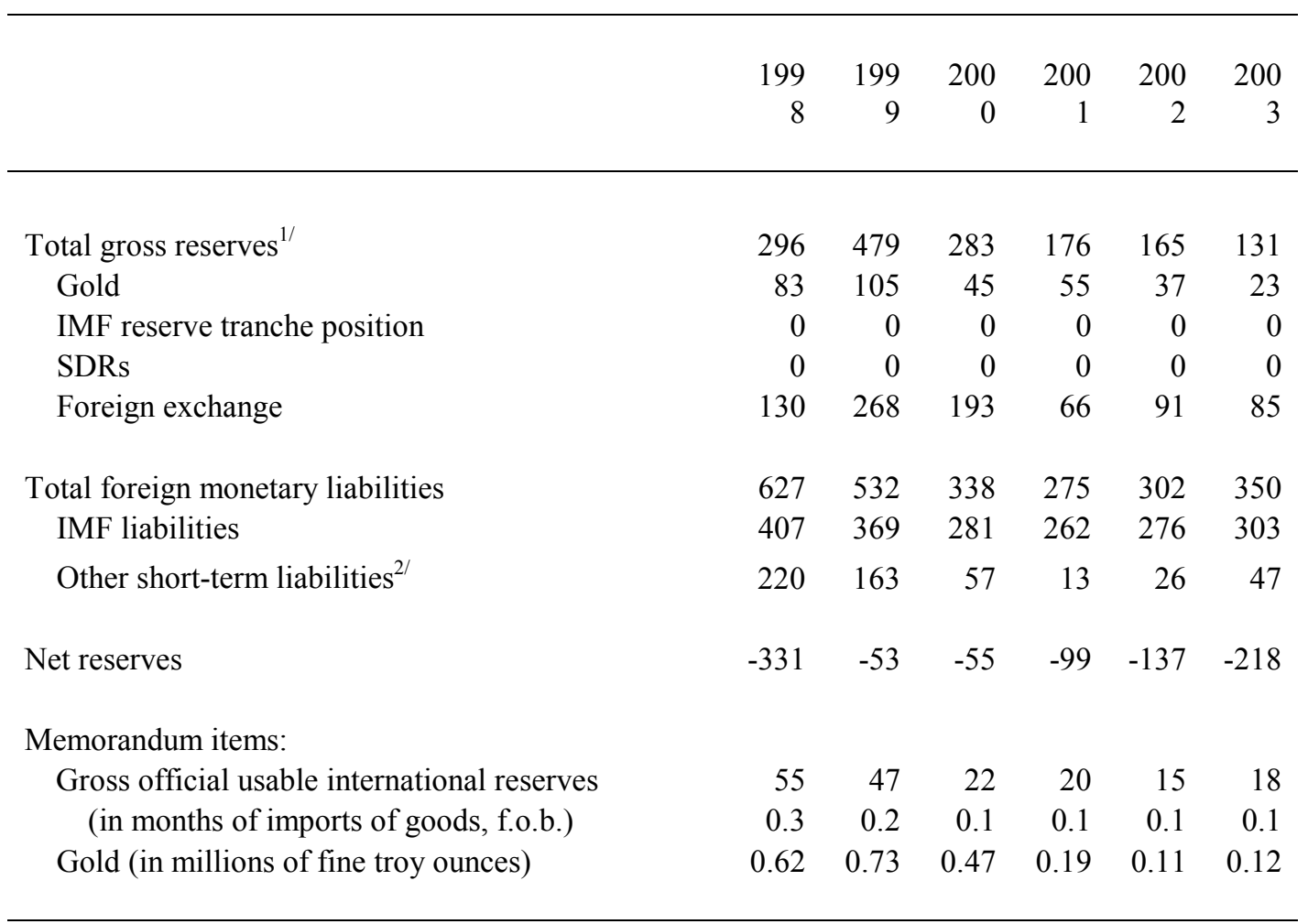

Source: Reserve Bank of Zimbabwe.

1/ Official gross reserves include pledged and illiquid assets.

2/ Includes open general import license (OGIL) short-term facility, Reserve Bank stand-by credits, foreign currency deposits held by residents, and foreign bank deposits. 
Table 38. Zimbabwe: External Debt Outstanding By Creditors, 1998-2003

\begin{tabular}{|c|c|c|c|c|c|c|}
\hline & 1998 & 1999 & 2000 & 2001 & 2002 & 2003 \\
\hline Public and publicly guaranteed debt (excl. arrears) & 3,785 & 3,556 & 3,112 & 2,664 & 2,594 & 2,545 \\
\hline Medium- and long-term debt (excl. arrears) & 3,553 & 3,324 & 2,880 & 2,432 & 2,362 & 2,313 \\
\hline Bilateral creditors & 1,238 & 1,141 & 1,031 & 926 & 977 & 1,019 \\
\hline Multilateral institutions & 1,904 & 1,829 & 1,581 & 1,320 & 1,229 & 1,170 \\
\hline IMF & 392 & 368 & 284 & 190 & 98 & 41 \\
\hline IBRD & 498 & 473 & 416 & 392 & 421 & 449 \\
\hline Others & 1,014 & 989 & 881 & 738 & 710 & 680 \\
\hline Private creditors & 410 & 353 & 267 & 186 & 156 & 124 \\
\hline Short-term debt (excl. arrears) & 232 & 232 & 232 & 232 & 232 & 232 \\
\hline Private debt (excl. arrears) & 672 & 642 & 498 & 377 & 256 & 157 \\
\hline Total debt (excl. arrears) & 4,457 & 4,198 & 3,610 & 3,042 & 2,850 & 2,702 \\
\hline Arrears on public and publicly guaranteed debt & 9 & 123 & 404 & 803 & 1,520 & 2,141 \\
\hline Medium- and long-term debt & 9 & 7 & 213 & 636 & 1,173 & 1,702 \\
\hline Bilateral creditors & 1 & 1 & 69 & 166 & 337 & 519 \\
\hline Multilateral institutions & 0 & 0 & 78 & 314 & 585 & 839 \\
\hline IMF & 0 & 0 & 0 & 76 & 169 & 262 \\
\hline IBRD & 0 & 0 & 0 & 5 & 0 & 62 \\
\hline Others & 0 & 0 & 78 & 233 & 416 & 515 \\
\hline Private creditors & 8 & 6 & 66 & 157 & 251 & 344 \\
\hline Short-term external debt & 0 & 7 & 44 & 57 & 65 & 71 \\
\hline Other public and publicly guaranteed arrears ${ }^{1 /}$ & 0 & 109 & 147 & 109 & 282 & 368 \\
\hline Total arrears & 9 & 123 & 404 & 803 & 1,520 & 2,141 \\
\hline Total debt & 4,466 & 4,321 & 4,014 & 3,845 & 4,370 & 4,843 \\
\hline Public and publicly guaranteed debt & 3,794 & 3,679 & 3,516 & 3,468 & 4,114 & 4,686 \\
\hline Private debt & 672 & 642 & 498 & 377 & 256 & 157 \\
\hline & \multicolumn{6}{|c|}{ (In percent of GDP) } \\
\hline Medium and long-term debt & 40.9 & 40.6 & 40.6 & 39.7 & 51.8 & 63.8 \\
\hline Of which: public and publicly guaranteed & 38.3 & 38.1 & 37.9 & 37.5 & 49.8 & 61.9 \\
\hline Short-term debt & 7.2 & 7.2 & 6.4 & 5.6 & 5.2 & 4.6 \\
\hline \multicolumn{7}{|l|}{ Memorandum item: } \\
\hline GDP (in millions of U.S. dollars) $)^{2 /}$ & 9,294 & 9,037 & 8,542 & 8,485 & 7,662 & 7,081 \\
\hline
\end{tabular}

Sources: Reserve Bank of Zimbabwe; and IMF staff estimates.

1/ Arrears on supplier credits and interest on arrears.

2/ Nominal U.S. dollar GDP adjusted for real growth and international inflation (1996 base year). 


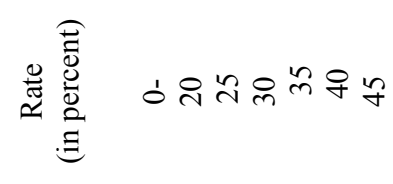

¿:\%:

i. 0.000

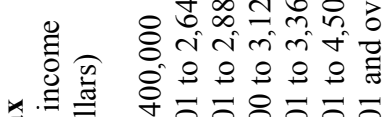

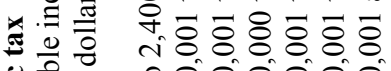

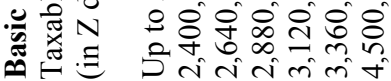

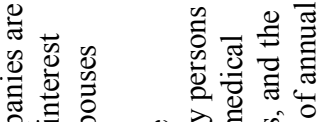

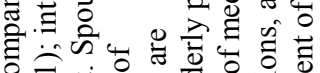

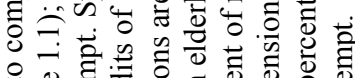

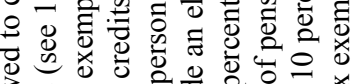

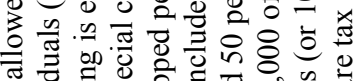

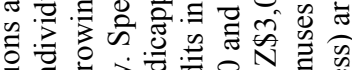

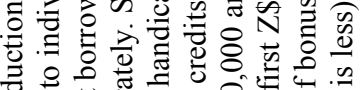

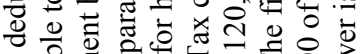
o

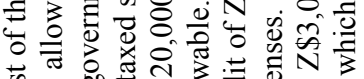

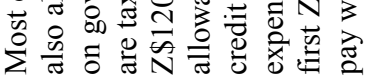

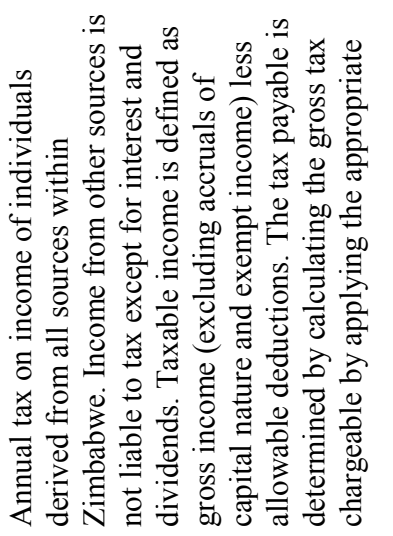

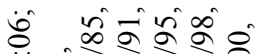

तुं क्षेत ते

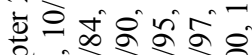

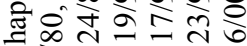

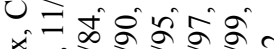

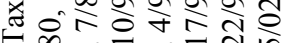

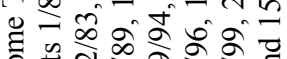

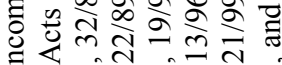

कि

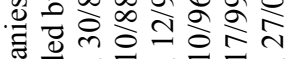

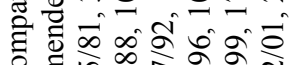

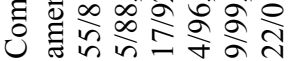

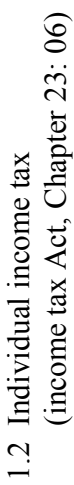


|)

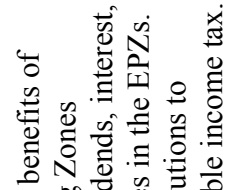

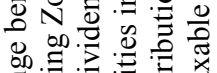

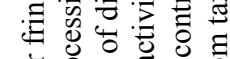

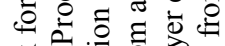

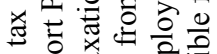

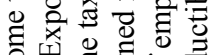

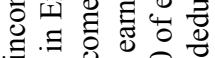

记

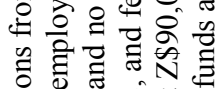

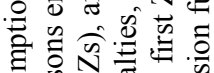

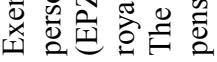

.

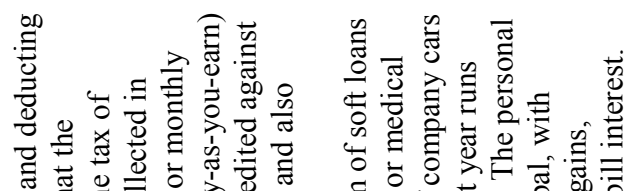

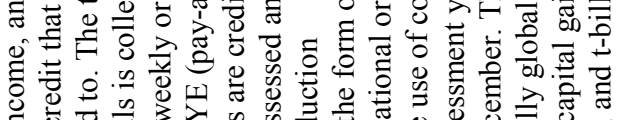

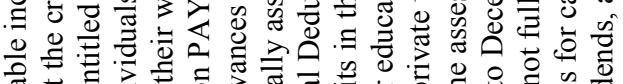

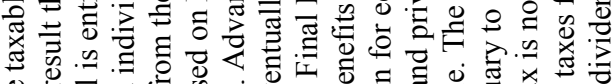

$-43-$

STATISTICAL APPENDIX
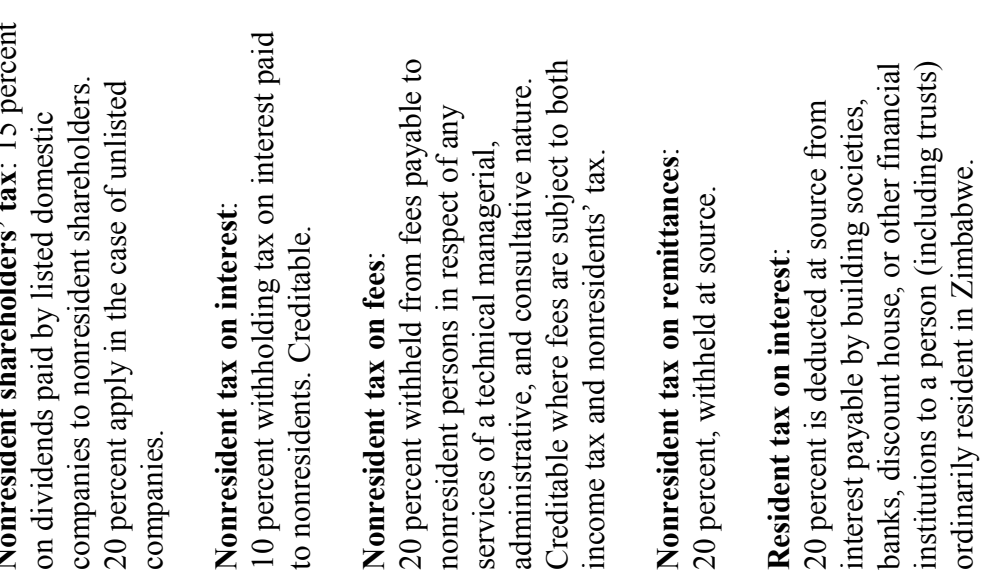

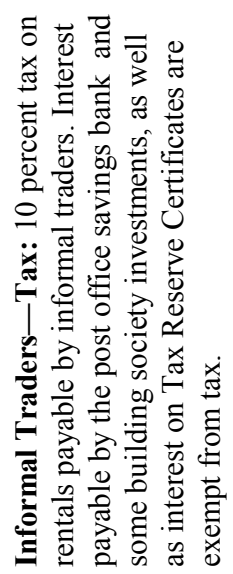

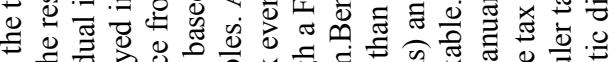

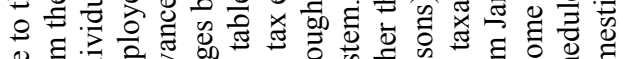

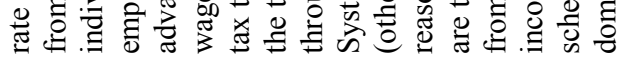



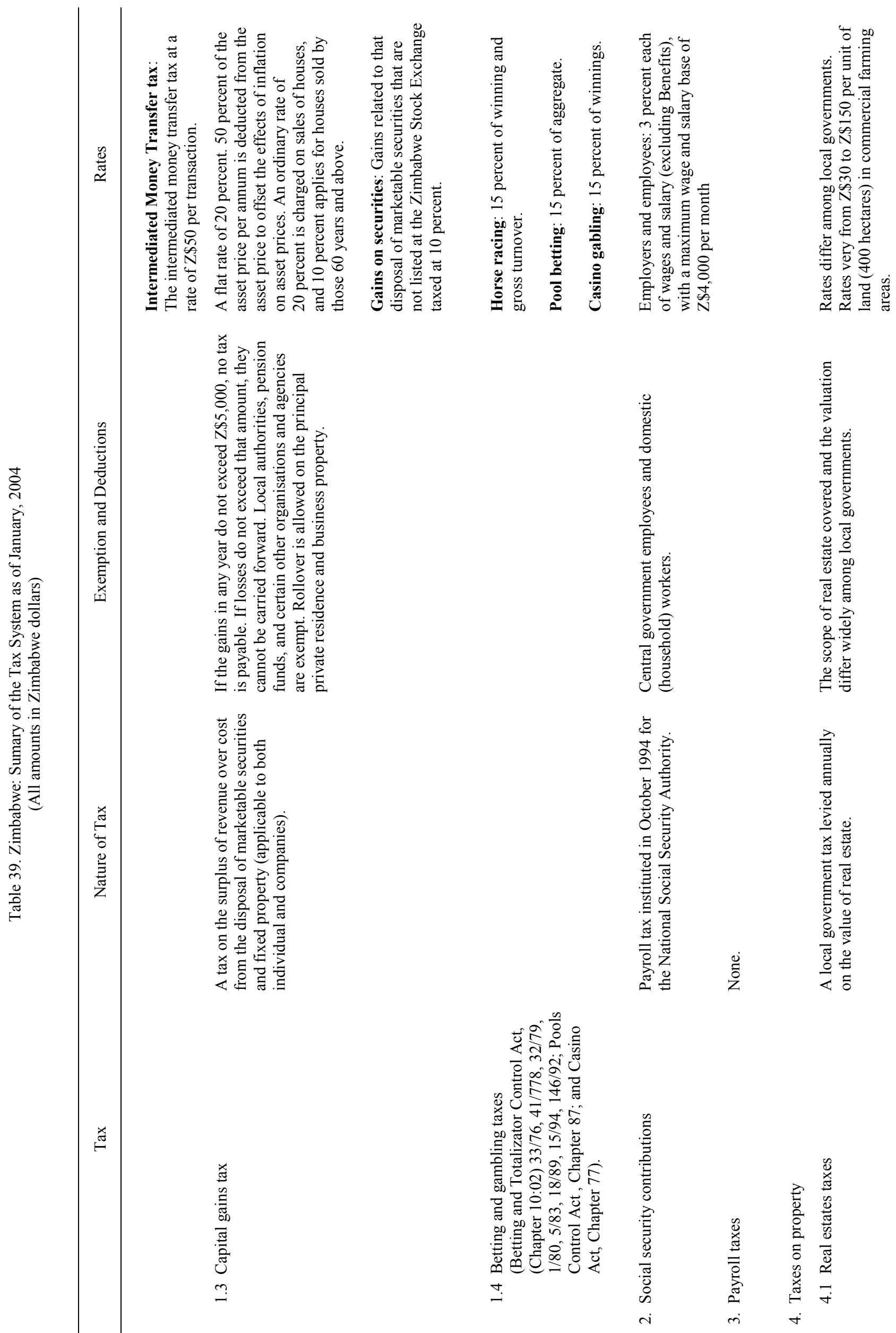


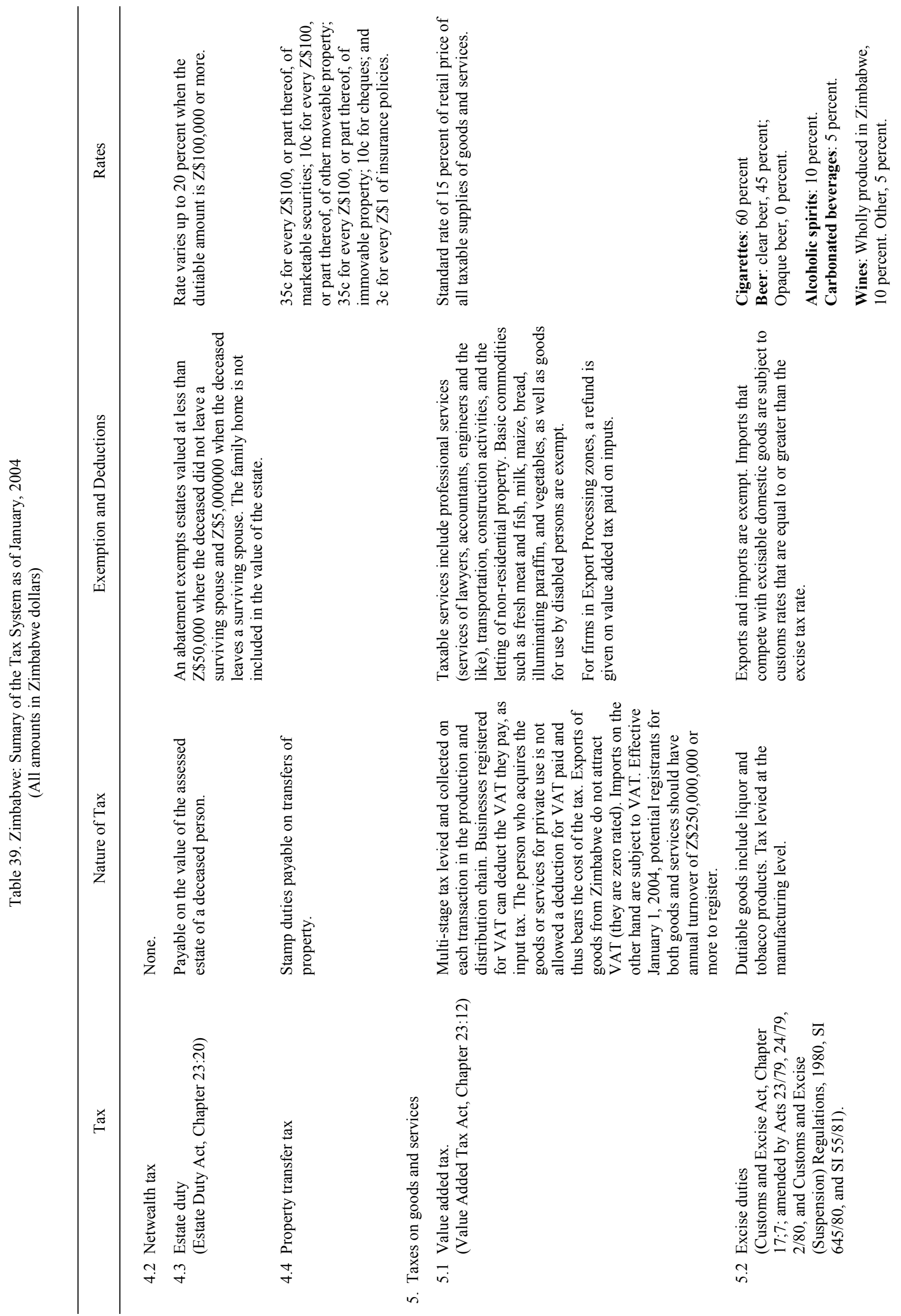




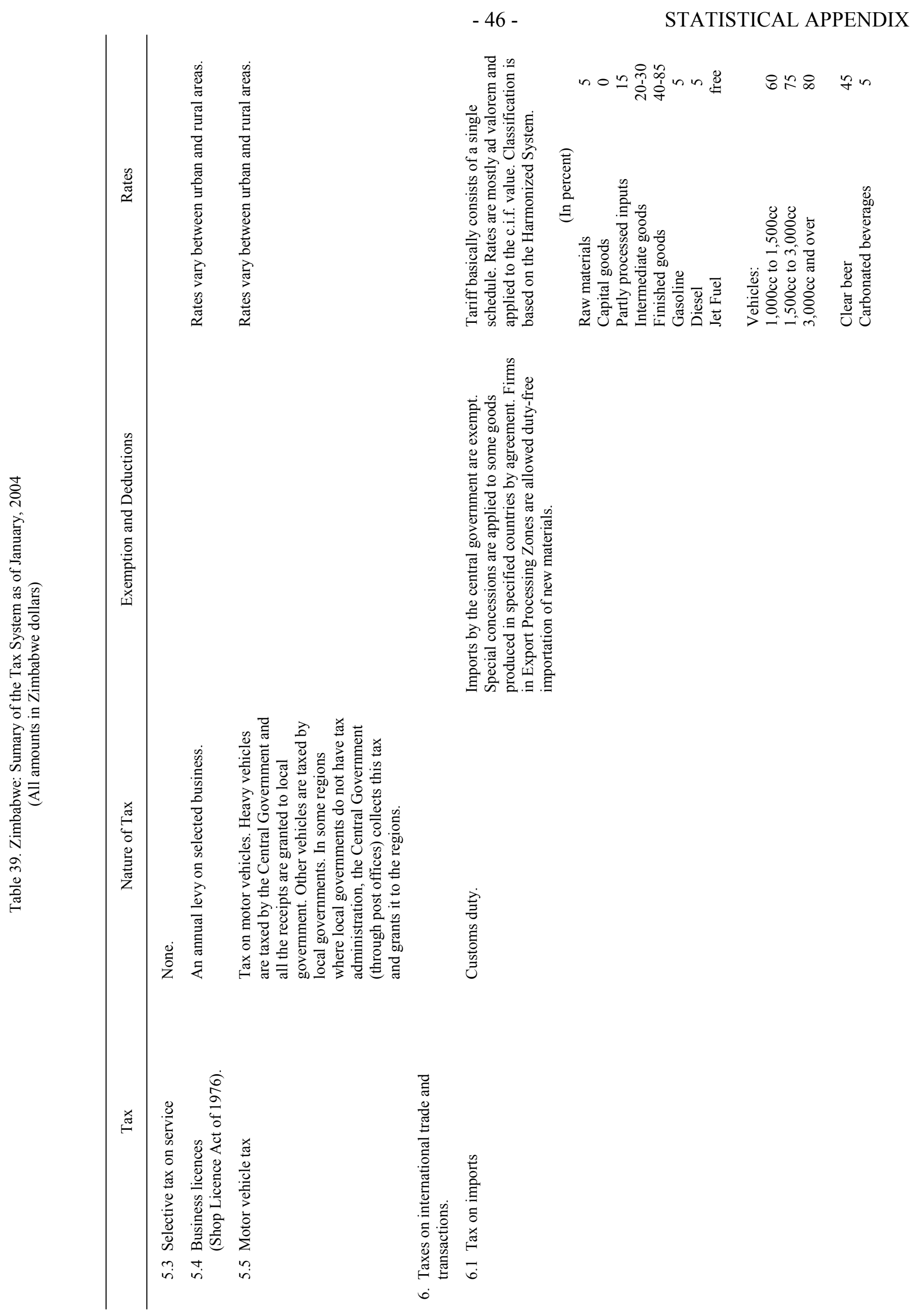




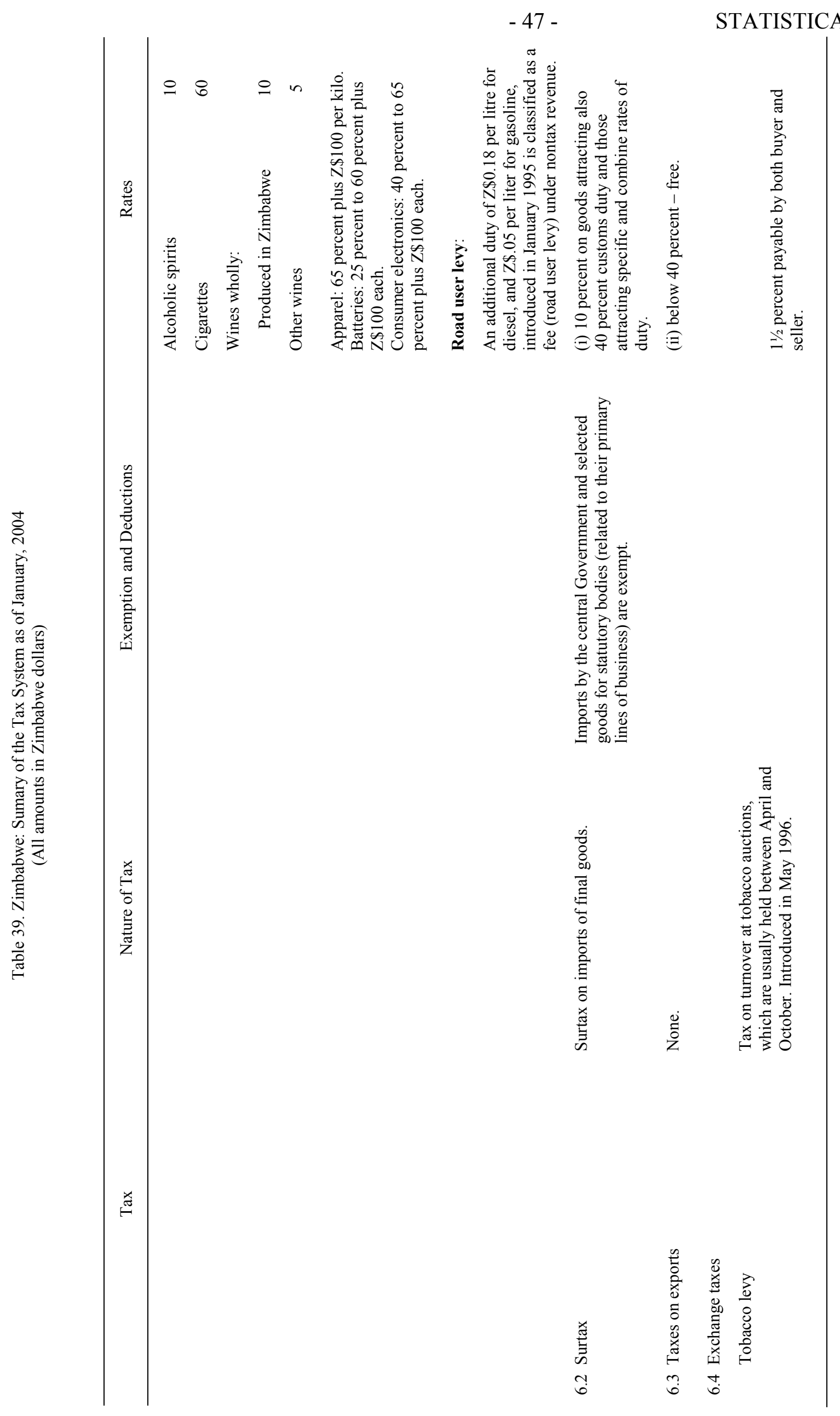

